\title{
Brownian Motion of Molecular Probes in Supercooled Liquids
}

\section{Citation}

Liu, Qihan, Shicheng Huang, and Zhigang Suo. 2015. "Brownian Motion of Molecular Probes in Supercooled Liquids." Physical Review Letters 114 (22) (June 4). doi:10.1103/ physrevlett.114.224301.

\section{Published Version}

doi:10.1103/PhysRevLett.114.224301

\section{Permanent link}

http://nrs.harvard.edu/urn-3:HUL.InstRepos:30403687

\section{Terms of Use}

This article was downloaded from Harvard University's DASH repository, and is made available under the terms and conditions applicable to Other Posted Material, as set forth at http:// nrs.harvard.edu/urn-3:HUL.InstRepos:dash.current.terms-of-use\#LAA

\section{Share Your Story}

The Harvard community has made this article openly available.

Please share how this access benefits you. Submit a story.

\section{Accessibility}




\title{
Joint Analysis of BICEP2/Keck Array and Planck Data
}

\author{
P. A. R. Ade et al. ${ }^{*}$ \\ (BICEP2/Keck and Planck Collaborations) \\ (Received 21 January 2015; published 9 March 2015)
}

\begin{abstract}
We report the results of a joint analysis of data from BICEP2/Keck Array and Planck. BICEP2 and Keck Array have observed the same approximately $400 \mathrm{deg}^{2}$ patch of sky centered on RA $0 \mathrm{~h}$, Dec. $-57.5^{\circ}$. The combined maps reach a depth of $57 \mathrm{nK}$ deg in Stokes $Q$ and $U$ in a band centered at $150 \mathrm{GHz}$. Planck has observed the full sky in polarization at seven frequencies from 30 to $353 \mathrm{GHz}$, but much less deeply in any given region $(1.2 \mu \mathrm{K}$ deg in $Q$ and $U$ at $143 \mathrm{GHz})$. We detect $150 \times 353$ cross-correlation in $B$ modes at high significance. We fit the single- and cross-frequency power spectra at frequencies $\geq 150 \mathrm{GHz}$ to a lensed- $\Lambda \mathrm{CDM}$ model that includes dust and a possible contribution from inflationary gravitational waves (as parametrized by the tensor-to-scalar ratio $r$ ), using a prior on the frequency spectral behavior of polarized dust emission from previous Planck analysis of other regions of the sky. We find strong evidence for dust and no statistically significant evidence for tensor modes. We probe various model variations and extensions, including adding a synchrotron component in combination with lower frequency data, and find that these make little difference to the $r$ constraint. Finally, we present an alternative analysis which is similar to a map-based cleaning of the dust contribution, and show that this gives similar constraints. The final result is expressed as a likelihood curve for $r$, and yields an upper limit $r_{0.05}<0.12$ at $95 \%$ confidence. Marginalizing over dust and $r$, lensing $B$ modes are detected at 7.0 $\sigma$ significance.
\end{abstract}

DOI: 10.1103/PhysRevLett.114.101301

PACS numbers: 98.70.Vc, 04.80.Nn, 95.85.Bh, 98.80.Es

\section{INTRODUCTION}

The cosmic microwave background (CMB) [1] is an essential source of information about all epochs of the Universe. In the past several decades, characterization of the temperature and polarization anisotropies of the CMB has helped to establish the standard cosmological model $(\Lambda \mathrm{CDM})$ and to measure its parameters to high precision (see, for example, Refs. [2,3]).

An extension to the standard big bang model, inflation, postulates a short period of exponential expansion in the very early Universe, naturally setting the initial conditions required by $\Lambda \mathrm{CDM}$, as well as solving a number of additional problems in standard cosmology. Inflation's basic predictions regarding the Universe's large-scale geometry and structure have been borne out by cosmological measurements to date (see Ref. [4] for a review). Inflation makes an additional prediction, the existence of a background of gravitational waves, or tensor mode perturbations [5-8]. At the recombination epoch, the inflationary gravitational waves (IGW) contribute to the anisotropy of the CMB in both total intensity and linear polarization. The amplitude of tensors is conventionally parametrized by $r$, the tensor-to-scalar ratio at a fiducial scale. Theoretical predictions of the value of $r$ cover a very wide range. Conversely, a measurement of $r$ can discriminate between models of inflation.

*Full author list given at the end of the article. Please cite as "BICEP2/Keck and Planck Collaborations."
Tensor modes produce a small increment in the temperature anisotropy power spectrum over the standard $\Lambda$ CDM scalar perturbations at multipoles $\ell \lesssim 60$; measuring this increment requires the large sky coverage traditionally achieved by space-based experiments, and an understanding of the other cosmological parameters. The effects of tensor perturbations on $B$-mode polarization is less ambiguous than on temperature or $E$-mode polarization over the range $\ell \lesssim 150$. The $B$-mode polarization signal produced by scalar perturbations is very small and is dominated by the weak lensing of $E$-mode polarization on small angular scales, making the detection of an IGW contribution possible [9-12].

Planck [13] was the third generation CMB space mission, which mapped the full sky in polarization in seven bands centered at frequencies from 30 to $353 \mathrm{GHz}$ to a resolution of 33 to 5 arc min [14,15]. The Planck Collaboration has published the best limit to date on tensor modes using CMB data alone [3]: $r_{0.002}<0.11$ (at 95\% confidence) using a combination of Planck, SPT, and ACT temperature data, plus WMAP polarization, although the Planck $r$ limit is model dependent, with running of the scalar spectral index or additional relativistic degrees of freedom being well-known degeneracies which allow larger values of $r$.

Interstellar dust grains produce thermal emission, the brightness of which increases rapidly from the $100-150 \mathrm{GHz}$ frequencies favored for CMB observations, becoming dominant at $\geq 350 \mathrm{GHz}$ even at high galactic latitude. The dust grains align with the Galactic magnetic 
field to produce emission with a degree of linear polarization [16]. The observed degree of polarization depends on the structure of the Galactic magnetic field along the line of sight, as well as the properties of the dust grains (see, for example, Refs. [17,18]). This polarized dust emission results in both $E$ mode and $B$ mode, and acts as a potential contaminant to a measurement of $r$. Galactic dust polarization was detected by Archeops [19] at $353 \mathrm{GHz}$ and by WMAP $[2,20]$ at $90 \mathrm{GHz}$.

BICEP2 was a specialized, low angular resolution experiment, which operated from the South Pole from 2010 to 2012, concentrating $150 \mathrm{GHz}$ sensitivity comparable to Planck on a roughly $1 \%$ patch of sky at high Galactic latitude [21]. The BICEP2 Collaboration published a highly significant detection of $B$-mode polarization in excess of the $r=0$ lensed- $\Lambda$ CDM expectation over the range $30<\ell<150$ in Ref. [22] (hereafter BK-I). Modest evidence against a thermal Galactic dust component dominating the observed signal was presented based on the cross spectrum against $100 \mathrm{GHz}$ maps from the previous BICEP1 experiment. The detected $B$-mode level was higher than that projected by several existing dust models $[23,24]$ although these did not claim any high degree of reliability.

The Planck survey released information on the structure of the dust polarization sky at intermediate latitudes [25], and the frequency dependence of the polarized dust emission at frequencies relevant to $\mathrm{CMB}$ studies [26]. Other papers argued that the BICEP2 region is significantly contaminated by dust $[27,28]$. Finally, Planck released information on dust polarization at high latitude [29] (hereafter PIP-XXX), and in particular examined a field centered on the BICEP2 region (but somewhat larger than it) finding a level of polarized dust emission at $353 \mathrm{GHz}$ sufficient to explain the $150 \mathrm{GHz}$ excess observed by BICEP2, although with relatively low signal-to-noise.

Keck Array is a system of BICEP2-like receivers also located at the South Pole. During the 2012 and 2013 seasons Keck Array observed the same field as BICEP2 in the same $150 \mathrm{GHz}$ frequency band [30] (hereafter BK-V). Combining the BICEP2 and Keck Array maps yields $Q$ and $U$ maps with rms noise of $57 \mathrm{nK}$ in nominal $1 \mathrm{deg}^{2}$ pixels - by far the deepest made to date.

In this Letter, we take cross spectra between the joint BICEP2/Keck maps and all the polarized bands of Planck. The structure is as follows. In Sec. II we describe the preparation of the input maps, the expectations for dust, and the power spectrum results. In Sec. III the main multifrequency cross-spectrum likelihood method is introduced and applied to the data, and a number of variations from the selected fiducial analysis are explored. Section IV describes validation tests using simulations as well as an alternate likelihood. In Sec. V we investigate whether there could be decorrelation between the Planck and BICEP2/Keck maps due to the astrophysics of dust and/or instrumental effects. Finally we conclude in Sec. VI.

\section{MAPS TO POWER SPECTRA}

\section{A. Maps and preparation}

We primarily use the BICEP2/Keck combined maps, as described in BK-V. We also use the BICEP2-only and Keck-only maps as a cross-check. The Planck maps used for cross-correlation with BICEP2/Keck are the fullmission polarized maps from the PR2 Planck science release [31,32], a subset of which was presented in PIP-XXX. We compute Planck single-frequency spectra as the cross-power spectra of two data-split maps, in which the data are split into two subsets with independent noise. We consider three data split maps: (i) detector-set maps, where the detectors at a given frequency are divided into two groups, (ii) yearly maps, where the data from the first and second years of observations are used for the two maps, and (iii) half-ring maps, where the data from each pointing period is divided in halves. To evaluate uncertainties due to Planck instrumental noise, we use 500 noise simulations of each map; these are the standard set of time-ordered data noise simulations projected into sky maps (the full focal plane 8 (FFP8) simulations defined in Ref. [33]).

While the Planck maps are filtered only by the instrument beam (the effective beam defined in Refs. [34] and [35]), the BICEP2/Keck maps are in addition filtered due to the observation strategy and analysis process. In particular, large angular scales are suppressed anisotropically in the BICEP2/Keck mapmaking process to avoid atmospheric and ground-fixed contamination; this suppression is corrected in the power spectrum estimate. In order to facilitate comparison, we therefore prepare "Planck as seen by BICEP2/Keck" maps. In the first step we use the ANAFAST, ALTERALM, and SYNFAST routines from the HEALPix [36] package [37] to resmooth the Planck maps with the BICEP2/Keck beam profile, assuming azimuthal symmetry of the beam. The coordinate rotation from Galactic to celestial coordinates of the $T, Q$, and $U$ maps is performed using the ALTERALM routine in the HEALPix package. The sign of the Stokes $U$ map is flipped to convert from the HEALPix to the IAU polarization convention. Next we pass these through the "observing" matrix $R$, described in Sect. VI. B of BK-I, to produce maps that include the filtering of modes occurring in the data processing pipeline (including polynominal filtering and scan-synchronous template removal, plus deprojection of beam systematics).

Figure 1 shows the resmoothed Planck $353 \mathrm{GHz} T, Q$, and $U$ maps before and after filtering. In both cases the BICEP2/Keck inverse variance apodization mask has been applied. This figure emphasizes the need to account for the filtering before any comparison of maps is attempted, either qualitative or quantitative.

\section{B. Expected spatial and frequency spectra of dust}

Before examining the power spectra it is useful to review expectations for the spatial and frequency spectra of dust. 


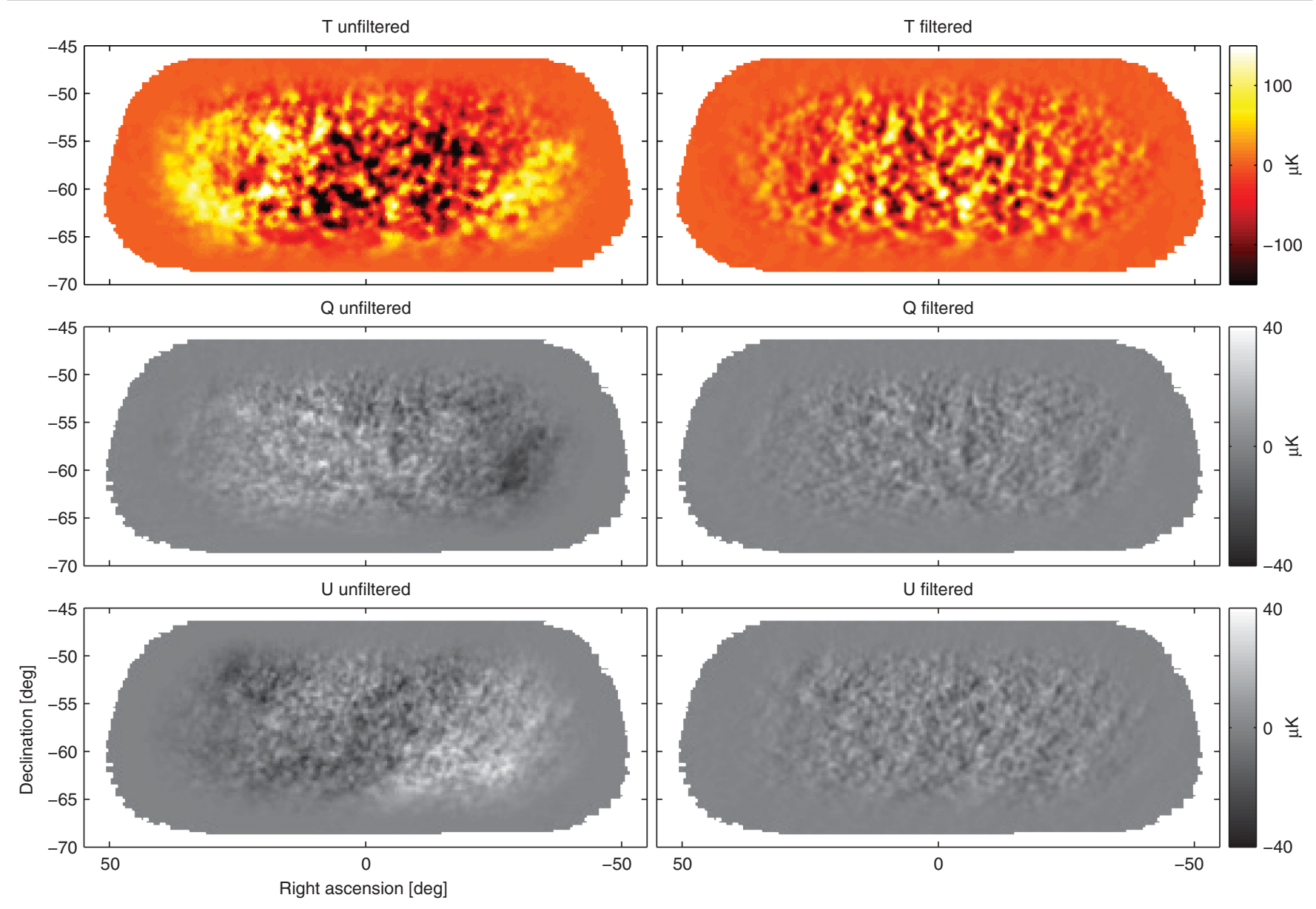

FIG. 1 (color). Planck $353 \mathrm{GHz} T$, $Q$, and $U$ maps before (left) and after (right) the application of BICEP2/Keck filtering. In both cases the maps have been multiplied by the BICEP2/Keck apodization mask. The Planck maps are presmoothed to the BICEP2/Keck beam profile and have the mean value subtracted. The filtering, in particular the third order polynominal subtraction to suppress atmospheric pickup, removes large-angular scale signal along the BICEP2/Keck scanning direction (parallel to the right ascension direction in the maps here).

Figure 2 of PIP-XXX shows that the dust $B B$ (and $E E$ ) angular power spectra are well fit by a simple power law $\mathcal{D}_{\ell} \propto \ell^{-0.42}$, where $\mathcal{D}_{\ell}=C_{\ell} \ell(\ell+1) / 2 \pi$, when averaging over large regions of sky outside of the Galactic plane. Section V B of the same paper states that there is no evidence for departure from this behavior for $1 \%$ sky patches, although the signal-tonoise ratio is low for some regions. Presumably we expect greater fluctuation from the mean behavior than would be expected for a Gaussian random field.

The spectral energy distribution (SED) of dust polarization was measured in Ref. [26] for 400 patches with $10^{\circ}$ radius at intermediate Galactic latitudes. Figure 10 of this reference shows empirically that the mean polarized dust SED is described by a simple modified blackbody spectrum with $T_{d}=19.6 \mathrm{~K}$ and $\beta_{d}=1.59 \pm 0.17$ to within an accuracy of a few percent over the frequency range 100 $353 \mathrm{GHz}$. Within this frequency range variations in the two parameters are highly degenerate and the choice is made to hold $T_{d}$ fixed at the value obtained from a fit to the SED The power spectrum estimation proceeds exactly as in
BK-I, including the matrix based purification operation to prevent $E$ to $B$ mixing. Figure 2 shows the results for BICEP2/Keck and Planck $353 \mathrm{GHz}$ for TT, TE, EE, and $B B$. In all cases the error bars are the standard deviations of lensed- $\Lambda \mathrm{CDM}+$ noise simulations [38] and hence contain no sample variance on any other component. The results in the left column are autospectra, identical to those given in BK-I and BK-V - these spectra are consistent with lensed $\Lambda \mathrm{CDM}+$ noise except for the excess in $B B$ for $\ell<200$.

The right column of Fig. 2 shows cross spectra between two halves of the Planck $353 \mathrm{GHz}$ data set, with three different splits shown. The Planck Collaboration prefers the use of cross spectra even at a single frequency to gain additional immunity to systematics and to avoid the need to noise debias autospectra. The $T T$ spectrum is higher than $\Lambda \mathrm{CDM}$ around $\ell=200-$ presumably due to a dust contribution. The $E E$ and $B B$ spectra are noisy, but both appear to show an excess over $\Lambda$ CDM for $\ell<150$ - again presumably due to dust. We note that these spectra do not appear to follow the power-law expectation mentioned in Sec. II B, but we emphasize that the error bars 

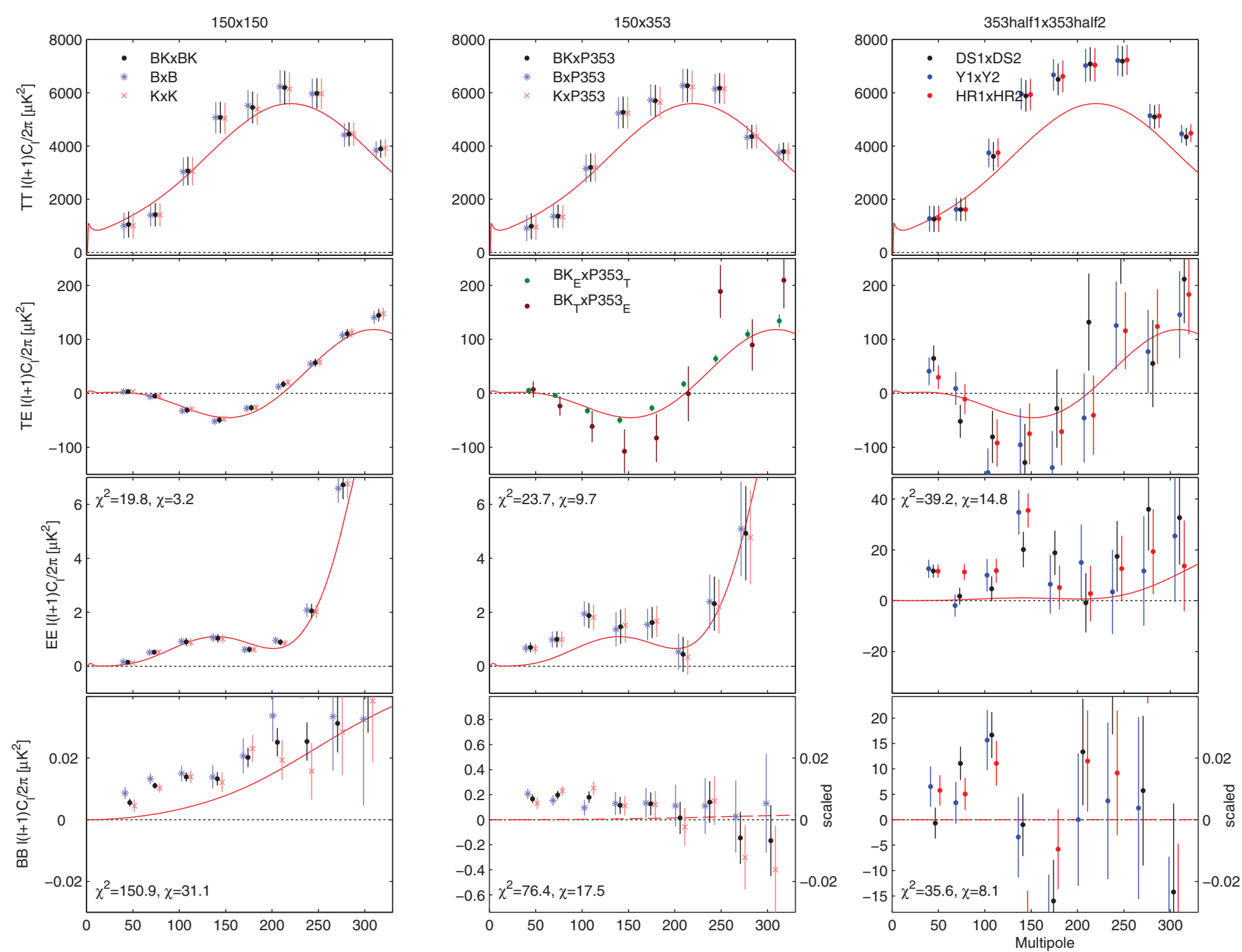

FIG. 2 (color). Single- and cross-frequency spectra between BICEP2/Keck maps at $150 \mathrm{GHz}$ and Planck maps at $353 \mathrm{GHz}$. The red curves show the lensed- $\Lambda \mathrm{CDM}$ expectations. The left column shows single-frequency spectra of the BICEP2, Keck Array and combined BICEP2/Keck maps. The BICEP2 spectra are identical to those in BK-I, while the Keck Array and combined are as given in BK-V. The center column shows cross-frequency spectra between BICEP2/Keck maps and Planck $353 \mathrm{GHz}$ maps. The right column shows Planck $353 \mathrm{GHz}$ data-split cross spectra. In all cases the error bars are the standard deviations of lensed- $\Lambda$ CDM + noise simulations and hence contain no sample variance on any other component. For $E E$ and $B B$ the $\chi^{2}$ and $\chi$ (sum of deviations) versus lensed $\Lambda C D M$ for the nine band powers shown is marked at upper and lower left (for the combined BICEP2/Keck points and DS $1 \times \mathrm{DS} 2$, respectively). In the bottom row (for $B B$ ) the center and right panels have a scaling applied such that signal from dust with the fiducial frequency spectrum would produce signal with the same apparent amplitude as in the $150 \mathrm{GHz}$ panel on the left (as indicated by the right-side $y$ axes). We see from the significant excess apparent in the bottom center panel that a substantial amount of the signal detected at $150 \mathrm{GHz}$ by BICEP2 and Keck Array indeed appears to be due to dust.

contain no sample variance on any dust component (Gaussian or otherwise).

The center column of Fig. 2 shows cross spectra between BICEP2/Keck and Planck maps. For $T E$ one can use the $T$ modes from BICEP2 and the $E$ modes from Planck or vice versa and both options are shown. Since the $T$ modes are very similar between the two experiments, these $T E$ spectra look similar to the single-experiment $T E$ spectrum which shares the $E$ modes. The $E E$ and $B B$ cross spectra are the most interesting - there appears to be a highly significant detection of correlated $B$-mode power between 150 and
$353 \mathrm{GHz}$, with the pattern being much brighter at 353, consistent with the expectation from dust. We also see hints of detection in the $E E$ spectrum-while dust $E$ modes are subdominant to the cosmological signal at $150 \mathrm{GHz}$, the weak dust contribution enhances the BK150 $\times$ P353 cross spectrum at $\ell \approx 100$.

The polarized dust SED model mentioned in Sec. II B implies that dust emission is approximately 25 times brighter in the Planck $353 \mathrm{GHz}$ band than it is in the BICEP2/Keck $150 \mathrm{GHz}$ band (integrating appropriately over the instrumental bandpasses). The expectation for a 

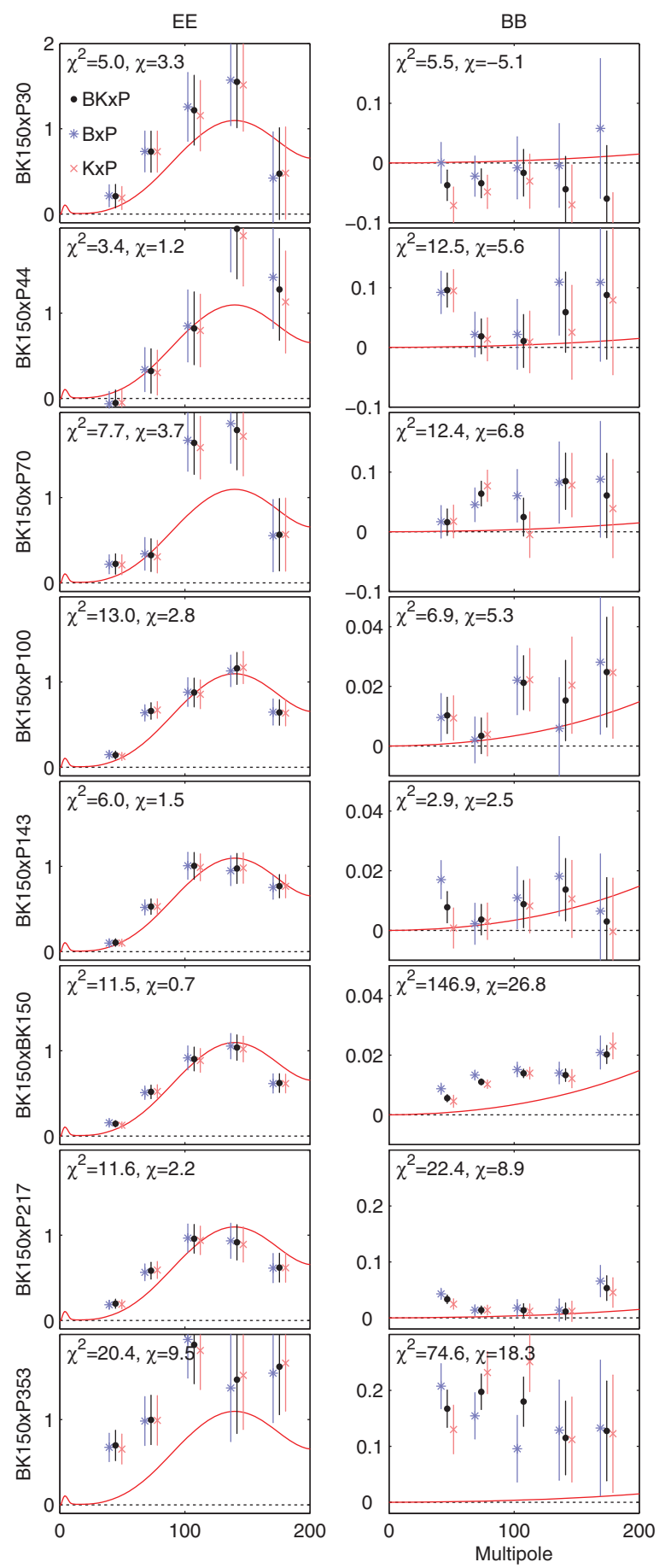

FIG. 3 (color). $\quad E E$ (left column) and $B B$ (right column) cross spectra between BICEP2/Keck maps and all of the polarized frequencies of Planck. In all cases the quantity plotted is $\ell(\ell+$ 1) $C_{l} / 2 \pi$ in units of $\mu \mathrm{K}_{\mathrm{CMB}}^{2}$, and the red curves show the lensed$\Lambda C D M$ expectations. The error bars are the standard deviations of lensed- $\Lambda \mathrm{CDM}+$ noise simulations and hence contain no sample variance on any other component. Also note that the $y$-axis scales differ from panel to panel in the right column. The $\chi^{2}$ and $\chi$ (sum of deviations) versus lensed $\Lambda \mathrm{CDM}$ for the five band powers shown is marked at upper left. There are no additional strong detections of deviation from lensed $\Lambda C D M$ over those already shown in Fig. 2 although BK150 $\times$ P217 shows some evidence of excess. dust-dominated spectrum is thus that the $\mathrm{BK} 150 \times \mathrm{P} 353$ cross spectrum should have an amplitude 25 times that of $\mathrm{BK} 150 \times \mathrm{BK} 150$, and P353 $\times \mathrm{P} 353$ should be 25 times higher again. The $y$-axis scaling in the bottom row of Fig. 2 has been adjusted so that a dust signal obeying this rule will have equal apparent amplitude in each panel. We see that a substantial amount of the BK150 $\times$ BK150 signal indeed appears to be due to dust.

To make a rough estimate of the significance of deviation from lensed $\Lambda \mathrm{CDM}$, we calculate $\chi^{2}$ and $\chi$ (sum of deviations) for each of the $E E$ and $B B$ spectra and show these in Fig. 2. For the nine band powers used the expectation value (standard deviation) for $\chi^{2}$ and $\chi$ are 9 (4.2) and 0 (3), respectively. We see that BK150 $\times$ BK150 and $\mathrm{BK} 150 \times \mathrm{P} 353$ are highly significant in $B B$, while $\mathrm{P} 353 \times \mathrm{P} 353$ has modest significance in both $E E$ and $B B$.

Figure 3 shows $E E$ and $B B$ cross spectra between BICEP2/Keck and all of the polarized frequencies of Planck (also including the BICEP2/Keck autospectra). For the five band powers shown the expectation value (standard deviation) for $\chi^{2}$ and $\chi$ are 5 (3.1) and 0 (2.2), respectively. As already noted, the BK150 $\times$ BK150 and BK150 $\times$ P353 $B B$ spectra show highly significant excesses. Additionally, there is evidence for excess $B B$ in $\mathrm{BK} 150 \times \mathrm{P} 217$ spectrum, and for excess $E E$ in $\mathrm{BK} 150 \times \mathrm{P} 353$. The other spectra in Fig. 3 show no strong evidence for excess, although we note that only one of the $\chi$ values is negative. There is weak evidence for excess in the BK150 $\times$ P70 $B B$ spectrum but none in $\mathrm{BK} 150 \times \mathrm{P} 30$ so this is presumably just a noise fluctuation.

There are a large number of additional Planck-only spectra, which are not plotted here. The noise on these is large and all are consistent with $\Lambda \mathrm{CDM}$, with the possible exception of P217 $\times$ P353, where modest evidence for an excess is seen in both $E E$ and $B B$ (see, e.g., Fig. 10 of PIP-XXX).

\section{Consistency of BICEP2 and Keck Array spectra}

The $B B$ autospectra for BICEP2 and Keck Array in the lower left panel of Fig. 2 appear to differ by more than might be expected, given that the BICEP 2 and Keck maps cover almost exactly the same region of sky. However, the error bars in this figure are the standard deviations of lensed- $\Lambda \mathrm{CDM}+$ noise simulations; while the signal is largely common between the two experiments the noise is not, and the signal-noise cross terms produce substantial additional fluctuation of the difference. The correct way to quantify this is to compare the difference of the real data to the pairwise differences of simulations, using common input skies that have power similar to that observed in the real data. This was done in Sec. 8 of BK-V and the BICEP2 and Keck maps were shown to be statistically compatible. In an analogous manner we can also ask if the B150 $\times$ P353 and $\mathrm{K} 150 \times \mathrm{P} 353 \mathrm{BB}$ cross spectra shown in the bottom middle panel of Fig. 2 are compatible. Figure 4 shows the results. We calculate the $\chi^{2}$ and $\chi$ statistics on these 


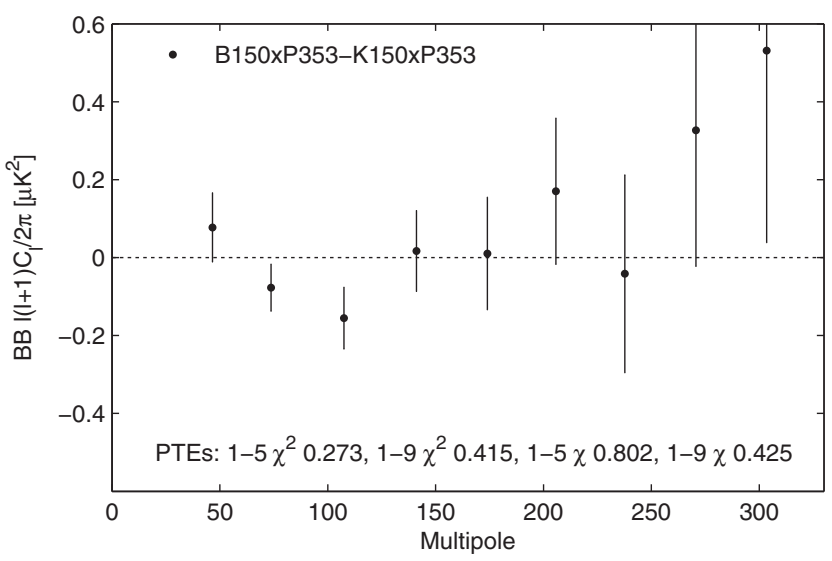

FIG. 4. Differences of $\mathrm{B} 150 \times \mathrm{P} 353$ and $\mathrm{K} 150 \times \mathrm{P} 353 B B$ cross spectra. The error bars are the standard deviations of the pairwise differences of signal + noise simulations that share common input skies. The probability to exceed the observed values of $\chi^{2}$ and $\chi$ statistics, as evaluated against the simulations, is quoted for band-power ranges $1-5(20<\ell<200)$ and $1-9$ $(20<\ell<330)$. There is no evidence that these spectra are statistically incompatible.

difference spectra and compare to the simulated distributions exactly as in BK-V. The probability to exceed (PTE) the observed values is given in the figure for band powers $1-5(20<\ell<200)$ and $1-9(20<\ell<330)$. There is no evidence that these spectra are statistically incompatible.

\section{E. Alternative power spectrum estimation}

We check the reliability of the power spectrum estimation with an alternative pipeline. The filtered and purified Planck and BICEP2 maps used to make the spectra shown in Fig. 2 are transformed back into the HEALPix pixelization using cubic spline interpolation. The $B$-mode cross power is then computed with the XPOL [39] and PureCL [40] estimators. Figure 5 shows the difference between these alternative band powers and the standard band powers for the B150 $\times$ P353 BB cross spectrum. As in Fig. 4 the error bars are the standard deviations of pairwise differences of simulations, which share common input skies and have power similar to that observed in the real data. The agreement is not expected to be exact due to the differing band-power window functions, but the differences of the real band powers are consistent with those of the simulations.

\section{LIKELIHOOD ANALYSIS}

\section{A. Algorithm}

While it is conventional in plots like Fig. 2 to present band powers with symmetric error bars, it is important to appreciate that this is an approximation. The likelihood of an observed band power for a given model expectation value is generally an asymmetric function, which can be

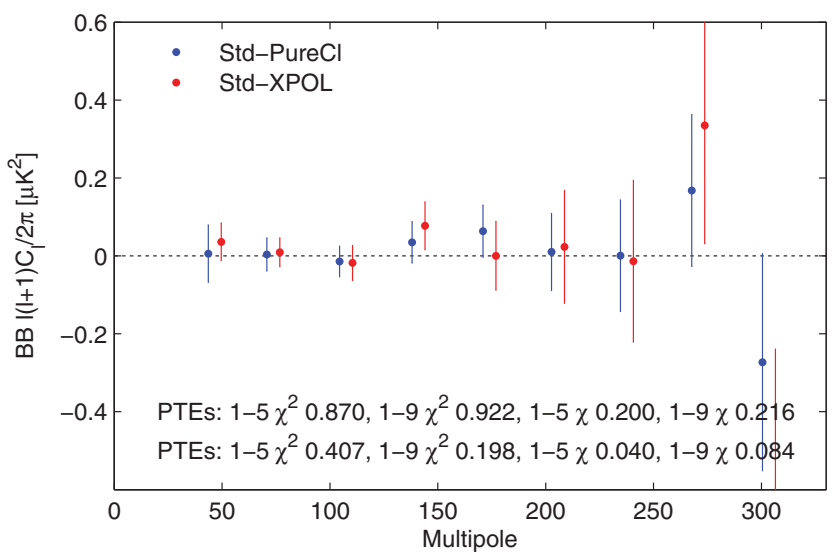

FIG. 5 (color). Differences of B150 $\times$ P353 BB cross spectra from the standard power spectrum estimator and alternate estimators. The error bars are the standard deviations of the pairwise differences of signal+noise simulations that share common input skies. The probability to exceed the observed values of $\chi^{2}$ and $\chi$ statistics, as evaluated against the simulations, is quoted for band-power ranges $1-5(20<\ell<200)$ and $1-9$ $(20<\ell<330)$. We see that the differences of the real spectra are consistent with the differences of the simulations.

computed given knowledge of the noise level(s). To compute the joint likelihood of an ensemble of measured band-power values it is, of course, necessary to consider their full covariance-this is especially important when using spectra taken at different frequencies on the same field, where the signal covariance can be very strong.

We compute the band-power covariance using full simulations of signal-cross-signal, noise-cross-noise, and signal-cross-noise. From these, we can construct the covariance matrix for a general model containing multiple signal components with any desired set of SEDs. When we do this we deliberately exclude terms whose expectation value is zero, in order to reduce noise in the resulting matrix due to the limited number of simulated realizations.

To compute the joint likelihood of the data for any given proposed model we use the Hamimeche-Lewis [41] approximation (HL; see Sec. 9.1 of Ref. [42] for mentation details). Here we extend the method to deal with single- and cross-frequency spectra, and the covariances thereof, in an analogous manner to the treatment of, for example, $T T, T E$, and $E E$ in the standard HL method. The HL formulation requires that the band-power covariance matrix be determined for only a single "fiducial model." We compute multidimensional grids of models explicitly and/or use Cosmomc [43] to sample the parameter space.

\section{B. Fiducial analysis}

As an extension of the simplest lensed- $\Lambda \mathrm{CDM}$ paradigm, we initially consider a two component model of IGW with amplitude $r$, plus dust with amplitude $A_{d}$ (specified at 

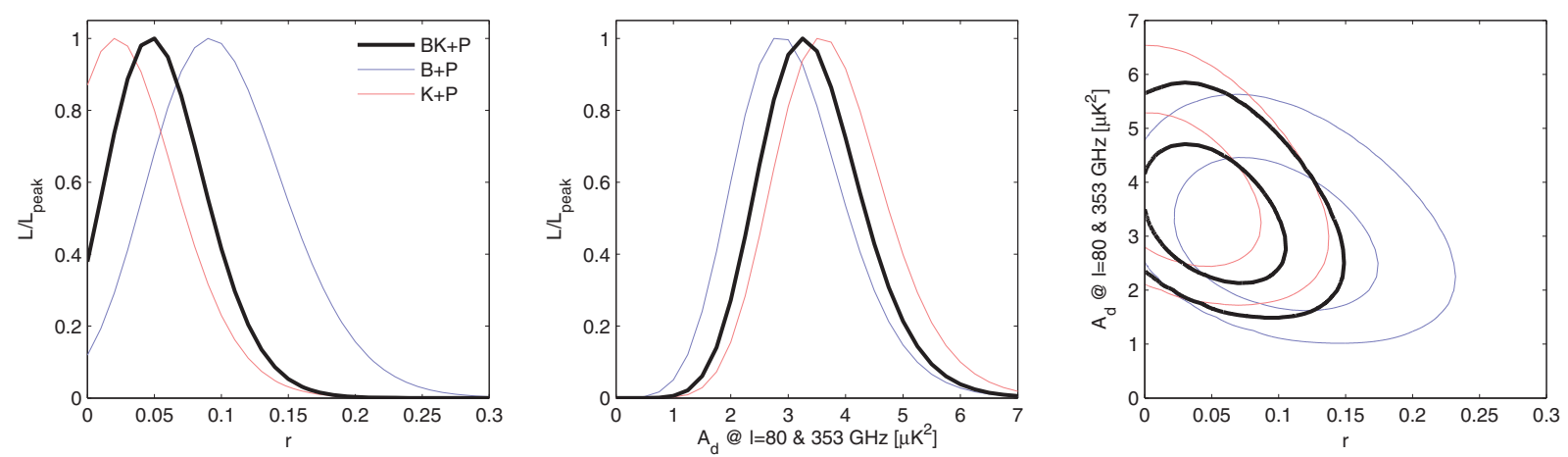

FIG. 6 (color). Likelihood results from a basic lensed- $\Lambda \mathrm{CDM}+r+$ dust model, fitting $B B$ autospectra and cross spectra taken between maps at $150 \mathrm{GHz}, 217$, and $353 \mathrm{GHz}$. The 217 and $353 \mathrm{GHz}$ maps come from Planck. The primary results (heavy black) use the $150 \mathrm{GHz}$ combined maps from BICEP2/Keck. Alternate curves (light blue and red) show how the results vary when the BICEP2 and Keck Array only maps are used. In all cases a Gaussian prior is placed on the dust frequency spectrum parameter $\beta_{d}=1.59 \pm 0.11$. In the right panel the two-dimensional contours enclose $68 \%$ and $95 \%$ of the total likelihood.

$353 \mathrm{GHz}$ and $\ell=80$ ). [Here we assume that the spectral index of the tensor modes $\left(n_{t}\right)$ is zero, and a scalar pivot scale of $0.05 \mathrm{Mpc}^{-1}$; all values of $r$ quoted in this Letter are $r_{0.05}$ unless noted otherwise.] Figure 6 shows the results of fitting such a model to $B B$ band powers taken between BICEP2/ Keck and the 217 and $353 \mathrm{GHz}$ bands of Planck, using band powers $1-5(20<\ell<200)$. For the Planck single-frequency case, the cross spectrum of detector sets $(\mathrm{DS} 1 \times \mathrm{DS} 2)$ is used, following PIP-XXX. The dust is modeled as a power law $\mathcal{D}_{\ell} \propto \ell^{-0.42}$, with free amplitude $A_{d}$ and scaling with frequency according to the modified blackbody model.

As discussed in Sec. II B the simple modified blackbody model is shown empirically in Ref. [26] to describe the mean polarized dust SED at mid-Galactic latitudes to an accuracy of a few percent over the frequency range 100$353 \mathrm{GHz}$, with variation of the $\beta_{d}$ parameter being sufficient to characterize the patch-to-patch variation. Since it is not possible to constrain $\beta_{d}$ using the BICEP2/Keck and Planck cross-spectral band powers alone a tight Gaussian prior $\beta_{d}=1.59 \pm 0.11$ is imposed, the uncertainty being scaled from the observed patch-topatch variation at intermediate Galactic latitudes in Ref. [26], as explained in PIP-XXX. This prior assumes that the SED of dust polarization at intermediate latitudes [26] applies to the high latitude BICEP2/Keck field. From dust astrophysics, we expect variations of the dust SED in intensity and polarization to be correlated [18]. We thus tested our assumption by measuring the $\beta_{d}$ of the dust total intensity in the BICEP2/Keck field using the template fitting analysis described in Ref. [44], and find the same value.

In Fig. 6 we see that the BICEP2 data produce an $r$ likelihood that peaks higher than that for the Keck Array data. This is because for $\ell<120$ the autospectrum B $150 \times$ $\mathrm{B} 150$ is higher than for $\mathrm{K} 150 \times \mathrm{K} 150$, while the cross spectrum $\mathrm{B} 150 \times \mathrm{P} 353$ is lower than $\mathrm{K} 150 \times \mathrm{P} 353$ (see
Fig. 2). However, recall that both pairs of spectra $B 150 \times$ $\mathrm{B} 150 / \mathrm{K} 150 \times \mathrm{K} 150$ and $\mathrm{B} 150 \times \mathrm{P} 353 / \mathrm{K} 150 \times \mathrm{P} 353$ have been shown to be consistent within noise fluctuation (see Sec. II D). In Sec. IV A these likelihood results are also found to be compatible. Given the consistency between the two experiments, the combined result gives the best available measurement of the sky.

The combined curves $(\mathrm{BK}+\mathrm{P})$ in the left and center panels of Fig. 6 yield the following results: $r=0.048_{-0.032}^{+0.035}$, $r<0.12$ at $95 \%$ confidence, and $A_{d}=3.3_{-0.8}^{+0.9}$. For $r$ the zero-to-peak likelihood ratio is 0.38 . Taking $\frac{1}{2}[1-$ $\left.f\left(-2 \log L_{0} / L_{\text {peak }}\right)\right]$, where $f$ is the $\chi^{2}$ cdf (for one degree of freedom), we estimate that the probability to get a number smaller than this is $8 \%$ if in fact $r=0$. For $A_{d}$ the zero-to-peak ratio is $1.8 \times 10^{-6}$ corresponding to a smallerthan probability of $1.4 \times 10^{-7}$, and a $5.1 \sigma$ detection of dust power.

The maximum likelihood model on the grid has parameters $r=0.05, A_{d}=3.30 \mu \mathrm{K}^{2}$ (and $\beta_{d}=1.6$ ). Computing the band-power covariance matrix for this model, we obtain a $\chi^{2}$ of 40.8 . Using 28 degrees of freedom -5 band powers times 6 spectra, minus 2 fit parameters (since $\beta_{d}$ is not really free)—gives a PTE of 0.06 . The largest contributions to $\chi^{2}$ come from the P353 $\times$ P353 spectrum shown in the lower right panel of Fig. 2.

\section{Variations from the fiducial data set and model}

We now investigate a number of variations from the fiducial analysis to see what difference these make to the constraint on $r$.

Choice of Planck single-frequency spectra: Switching the Planck single-frequency spectra to use one of the alternative data splits (yearly or half-ring instead of detector set) makes little difference (see Fig. 7).

Using only 150 and $353 \mathrm{GHz}$ : Dropping the spectra involving $217 \mathrm{GHz}$ from consideration also has little effect (see Fig. 7). 


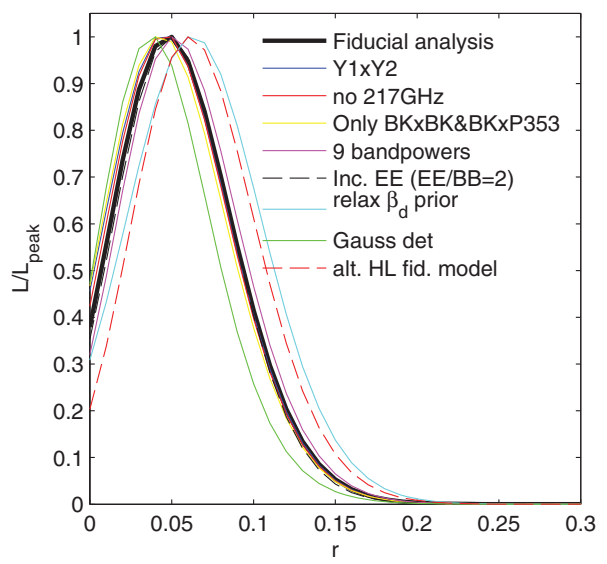

FIG. 7 (color). Likelihood results when varying the data sets used and the model priors-see Sec. III C for details.

Using only $\mathrm{BK} 150 \times \mathrm{BK} 150$ and $\mathrm{BK} 150 \times \mathrm{P} 353$ : Also excluding the $353 \mathrm{GHz}$ single-frequency spectrum from consideration makes little difference. The statistical weight of the BK150 $\times$ BK150 and BK150 $\times$ P353 spectra dominate (see Fig. 7).

Extending the band-power range: Going back to the base data set and extending the range of band powers considered to $1-9$ (corresponding to $20<\ell<330$ ) makes very little difference-the dominant statistical weight is with the lower band powers (see Fig. 7).

Including EE spectra: We can also include in the fits the EE spectra shown in Fig. 3. PIP-XXX (Figs. 5 and A.3) shows that the level of $E E$ from Galactic dust is on average around twice the level of $B B$. However, there are substantial variations in this ratio from sky patch to sky patch. Setting $E E / B B=2$ we find that the constraint on $A_{d}$ narrows, while the $r$ constraint changes little; this latter result is also shown in Fig. 7. The maximum likelihood model on the grid is unchanged and its $\chi^{2}$ PTE is acceptable.

Relaxing the $\beta_{d}$ prior: Relaxing the prior on the dust spectral index to $\beta_{d}=1.59 \pm 0.33$ pushes the peak of the $r$ constraint up (see Fig. 7). However, it is not clear if this looser prior is self-consistent; if the frequency spectral index varied significantly across the sky it would invalidate cross-spectral analysis, but there is strong evidence against such variation at high latitude, as explained in Sec. VA. Nevertheless, it is important to appreciate that the $r$ constraint curves shown in Fig. 6 shift left (right) when assuming a lower (higher) value of $\beta_{d}$. For $\beta_{d}=1.3 \pm 0.11$ the peak is at $r=0.021$ and for $\beta_{d}=1.9 \pm 0.11$ the peak is at $r=0.073$.

Varying the dust power spectrum shape: In the fiducial analysis the dust spatial power spectrum is assumed to be a power law with $\mathcal{D}_{\ell} \propto \ell^{-0.42}$. Marginalizing over spectral indices in the range -0.8 to 0 we find little change in the $r$ constraint (see also Sec. IV B for an alternate relaxation of the assumptions regarding the spatial properties of the dust pattern).

Using Gaussian determinant likelihood: The fiducial analysis uses the HL likelihood approximation, as described in Sec. III A. An alternative is to recompute the covariance matrix $\mathbf{C}$ at each point in parameter space and take $L=\operatorname{det}(\mathbf{C})^{-1 / 2} \exp \left[-\left(\mathbf{d}^{\mathrm{T}} \mathbf{C}^{-1} \mathbf{d}\right) / 2\right]$, where $\mathbf{d}$ is the deviation of the observed band powers from the model expectation values. This results in an $r$ constraint which peaks slightly lower, as shown in Fig. 7. Running both methods on the simulated realizations described in Sec. IV A, indicates that such a difference is not unexpected and that there may be a small systematic downward bias in the Gaussian determinant method.

Varying the HL fiducial model: As mentioned in Sec. III A the HL likelihood formulation requires that the expectation values and band-power covariance matrix be provided for a single "fiducial model" (not to be confused with the "fiducial analysis" of Sec. III B). The results from $\mathrm{HL}$ are supposed to be rather insensitive to the choice of this model, although preferably it should be close to reality. Normally, we use the lensed- $\Lambda \mathrm{CDM}+$ dust simulations described in Sec. IVA below. Switching this to lensed $\Lambda \mathrm{CDM}+r=0.2$ produces no change on average in the simulations, although it does cause any given realization to shift slightly - the change for the real data case is shown in Fig. 7.

Adding synchrotron: BK-I took the WMAP $K$-band (23 GHz) map, extrapolated it to $150 \mathrm{GHz}$ according to $\nu^{-3.3}$ (mean value within the BICEP2 field of the MCMC "Model f" spectral index map provided by WMAP [2]), and found a negligible predicted contribution $\left(r_{\text {sync, } 150}=0.0008 \pm 0.0041\right)$. Figure 3 does not offer strong motivation to reexamine this finding-the only significant detections of correlated $B B$ power are in the $\mathrm{BK} 150 \times \mathrm{P} 353$ and, to a lesser extent, BK150 $\times$ P217 spectra. However, here we proceed to a fit including all the polarized bands of Planck (as shown in Fig. 3) and adding a synchrotron component to the base lensed$\Lambda \mathrm{CDM}+$ noise $+r+$ dust model. We take synchrotron to have a power law spectrum $\mathcal{D}_{\ell} \propto \ell^{-0.6}$ [23], with free amplitude $A_{\text {sync }}$, where $A_{\text {sync }}$ is the amplitude at $\ell=80$ and at $150 \mathrm{GHz}$, and scaling with frequency according to $\nu^{-3.3}$. In such a scenario we can vary the degree of correlation that is assumed between the dust and synchrotron sky patterns. Figure 8 shows results for the uncorrelated and fully correlated cases. Marginalizing over $r$ and $A_{d}$ we find $A_{\text {sync }}<0.0003 \mu \mathrm{K}^{2}$ at $95 \%$ confidence for the uncorrelated case, and many times smaller for the correlated. This last is because once one has a detection of dust it effectively becomes a template for the synchrotron. This synchrotron limit is driven by the Planck $30 \mathrm{GHz}$ band-we obtain almost identical results when adding only this band, and a much softer limit when not including it. If we instead assume synchrotron scaling of $\nu^{-3.0}$ the limit on $A_{\text {sync }}$ is 

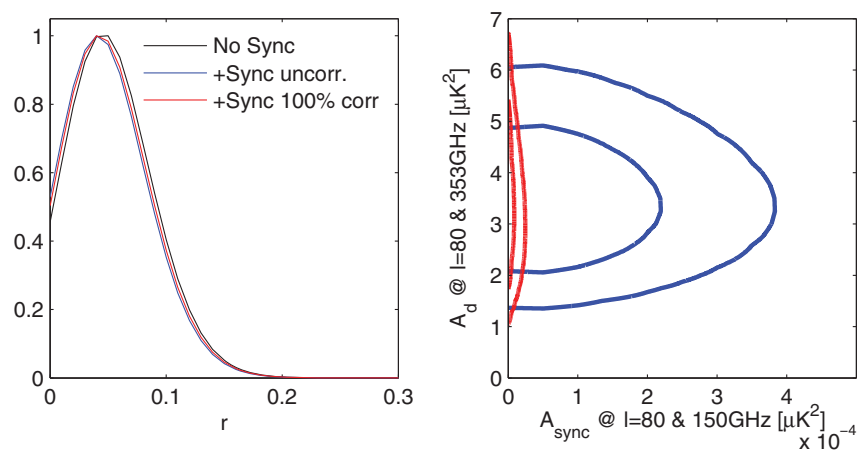

FIG. 8 (color). Likelihood results for a fit when adding the lower frequency bands of Planck, and extending the model to include a synchrotron component. The results for two different assumed degrees of correlation between the dust and synchrotron sky patterns are compared to those for the comparable model without synchrotron (see text for details).

approximately doubled for the uncorrelated case and reduced for the correlated. (Because the DS1 $\times$ DS2 data split is not available for the Planck LFI bands we switch to $\mathrm{Y} 1 \times \mathrm{Y} 2$ for this variant analysis, and so we compare to this case in Fig. 8 rather than the usual fiducial case.)

Varying lensing amplitude: In the fiducial analysis the amplitude of the lensing effect is held fixed at the $\Lambda$ CDM expectation $\left(A_{L}=1\right)$. Using their own and other data, the Planck Collaboration quote a limit on the amplitude of the lensing effect versus the $\Lambda \mathrm{CDM}$ expectation of $A_{L}=$ $0.99 \pm 0.05$ [3]. Allowing $A_{L}$ to float freely, and using all nine band powers, we obtain the results shown in Fig. 9-there is only weak degeneracy between $A_{L}$ and both $r$ and $A_{d}$. Marginalizing over $r$ and $A_{d}$ we find $A_{L}=1.13 \pm 0.18$ with a likelihood ratio between zero and peak of $3 \times 10^{-11}$. Using the expression given in Sec. III B this corresponds to a smaller-than probability of $2 \times 10^{-12}$, equivalent to a $7.0 \sigma$ detection of lensing in the $B B$ spectrum. We note this is the most significant to-date direct measurement of lensing in $B$ mode polarization.
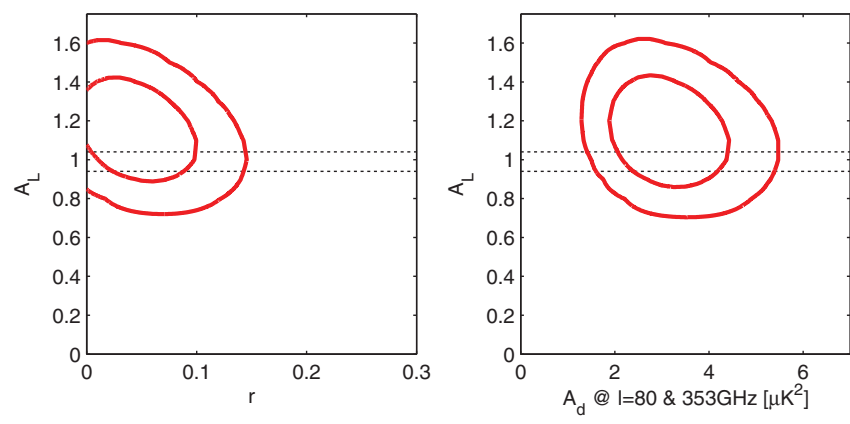

FIG. 9 (color). Likelihood results for a fit allowing the lensing scale factor $A_{L}$ to float freely and using all nine band powers. Marginalizing over $r$ and $A_{d}$, we find that $A_{L}=1.13 \pm 0.18$ and $A_{L}=0$ is ruled out with $7.0 \sigma$ significance.

\section{LIKELIHOOD VALIDATION}

\section{A. Validation with simulations}

We run the algorithm used in Sec. III B on ensembles of simulated realizations to check its performance. We first consider a model where $r=0$ and $A_{d}=3.6 \mu \mathrm{K}^{2}$, this latter being close to the value favored by the data in a dust-only scenario [45]. We generate Gaussian random realizations using the fiducial spatial power law $\mathcal{D}_{\ell} \propto \ell^{-0.42}$, scale these to the various frequency bands using the modified blackbody law with $T_{d}=19.6 \mathrm{~K}$ and $\beta_{d}=1.59$, and add to the usual realizations of lensed- $\Lambda \mathrm{CDM}+$ noise. Figure 10 shows some of the resulting $r$ and $A_{d}$ constraint curves, with the result for the real data from Fig. 6 overplotted. As expected, approximately $50 \%$ of the $r$ likelihoods peak above zero. The median 95\% upper limit is $r<0.075$. We find that $8 \%$ of the realizations have a ratio $L_{0} / L_{\text {peak }}$ less than the 0.38 observed in the real data, in agreement with the estimate in Sec. III B. Running these dust-only realizations for BICEP2 only and Keck Array only, we find that the shift in the maximum likelihood value of $r$ seen in the real data in Fig. 6 is exceeded in about $10 \%$ of the simulations.

The above simulations assume that the dust component follows on average the fiducial $\mathcal{D}_{\ell} \propto \ell^{-0.42}$ spatial power law, and fluctuates around it in a Gaussian manner. To obtain sample dust sky patterns that may deviate from this behavior in a way which better reflects reality, we take the prelaunch version of the Planck Sky Model (PSM; version 1.7.8 run in "simulation" mode) [24] evaluated in the Planck $353 \mathrm{GHz}$ band and pull out the same $352|b|>35^{\circ}$ partially overlapping regions used in PIP-XXX. We then scale these to the other bands and proceed as before. Some of the regions have dust power orders of magnitude higher than the real data and we cut them out (selecting 139 regions with peak $A_{d}<20 \mu \mathrm{K}^{2}$ ). Figure 11 presents the results. The $r$ likelihoods will broaden as the level of $A_{d}$ increases, and we should therefore not be surprised if the fraction of realizations peaking at a value higher than the real data is increased compared to the simulations with
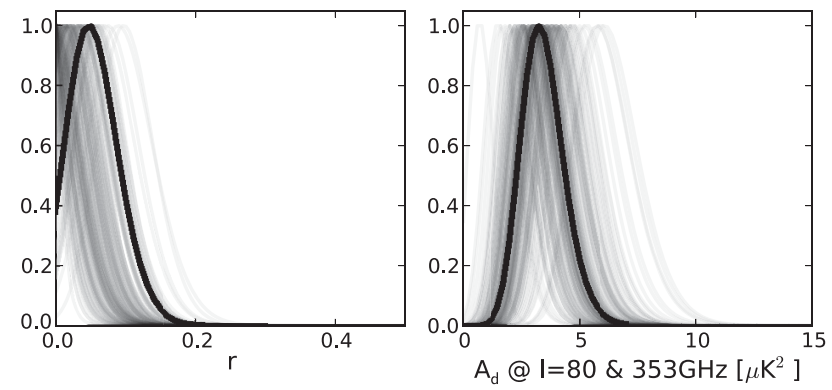

FIG. 10. Likelihoods for $r$ and $A_{d}$, using BICEP2/Keck and Planck, as plotted in Fig. 6, overplotted on constraints obtained from realizations of a lensed- $\Lambda \mathrm{CDM}+$ noise + dust model with dust power similar to that favored by the real data $\left(A_{d}=3.6 \mu \mathrm{K}^{2}\right)$. Half of the $r$ curves peak at zero as expected. 

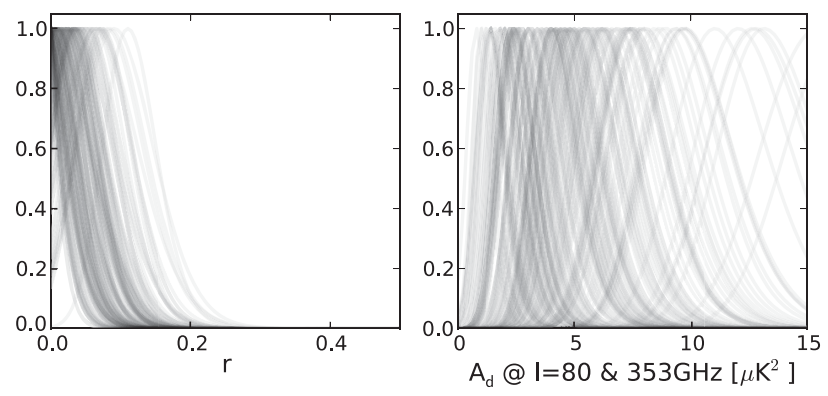

FIG. 11. Constraints obtained when adding dust realizations from the Planck Sky Model version 1.7.8 to the base lensed$\Lambda \mathrm{CDM}+$ noise simulations. (Curves for 139 regions with peak $A_{d}<20 \mu \mathrm{K}^{2}$ are plotted.) We see that the results for $r$ are unbiased in the presence of dust realizations which do not necessarily follow the $\ell^{-0.42}$ power law or have Gaussian fluctuations about it.

mean $A_{d}=3.6 \mu \mathrm{K}^{2}$. However, we still expect that on average $50 \%$ will peak above zero and approximately $8 \%$ will have an $L_{0} / L_{\text {peak }}$ ratio less than the 0.38 observed in the real data. In fact we find $57 \%$ and $7 \%$, respectively, consistent with the expected values. There is one realization which has a nominal (false) detection of nonzero $r$ of $3.3 \sigma$, although this turns out to also have one of the lowest $L_{0} / L_{\text {peak }}$ ratios in the Gaussian simulations shown in Fig. 10 (with which it shares the CMB and noise components), so this is apparently just a relatively unlikely fluctuation.

\section{B. Subtraction of scaled spectra}

As previously mentioned, the modified blackbody model predicts that dust emission is $4 \%$ as bright in the BICEP2 band as it is in the Planck $353 \mathrm{GHz}$ band. Therefore, taking the autospectra and cross spectra of the combined BICEP2/Keck maps and the Planck $353 \mathrm{GHz}$ maps, as shown in the bottom row of Fig. 2, and evaluating $(\mathrm{BK} \times \mathrm{BK}-\alpha \mathrm{BK} \times \mathrm{P}) /(1-\alpha)$, at $\alpha=\alpha_{\mathrm{fid}}$ cleans out the dust contribution (where $\alpha_{\mathrm{fid}}=0.04$ ). The upper panel of Fig. 12 shows the result.

As an alternative to the full likelihood analysis presented in Sec. III B, we can instead work with the differenced spectra from above, a method we denote the "cleaning" approach. If $\alpha_{\text {fid }}$ were the true value, the expectation value of this combination over $\mathrm{CMB}$ and noise would have no dust contribution. However, dust would still contribute to its variance, but only through its 2-point function. In practice, we do not know $\alpha$ perfectly, and this uncertainty needs to be accounted for in a likelihood constructed from the differenced spectra. Our approach is to treat the differenced spectra as a form of data compression, and to compute the expectation value as a function of $r, A_{d}$, and $\beta_{d}$ at each point in parameter space [the dust dependence enters for $\alpha\left(\beta_{d}\right) \neq \alpha_{\mathrm{fid}}$ ]. We use the method of Ref. [41], with a fiducial covariance matrix, to build a likelihood for
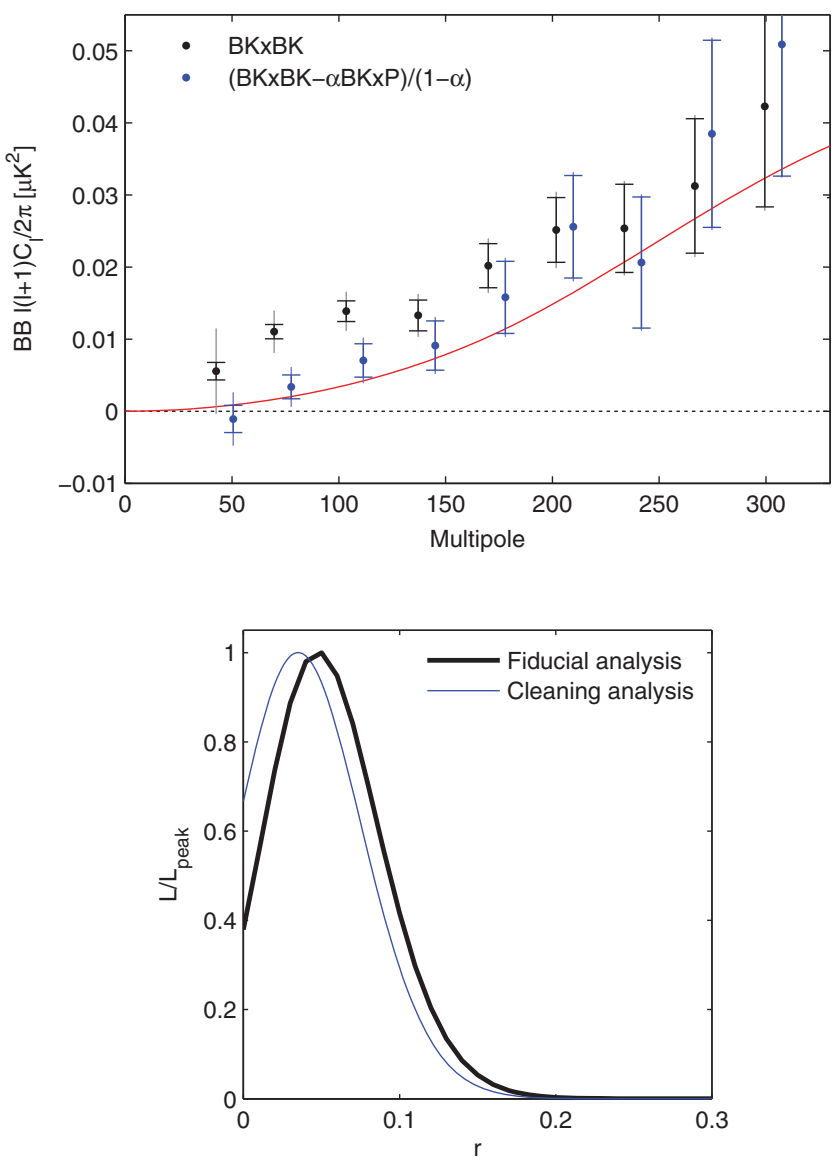

FIG. 12 (color). (Upper) BB spectrum of the BICEP2/Keck maps before and after subtraction of the dust contribution, estimated from the cross spectrum with Planck $353 \mathrm{GHz}$. The error bars are the standard deviations of simulations, which, in the latter case, have been scaled and combined in the same way. The inner error bars are from lensed- $\Lambda \mathrm{CDM}+$ noise simulations as in the previous plots, while the outer error bars are from the lensed- $\Lambda \mathrm{CDM}+$ noise + dust simulations. The red curve shows the lensed- $\Lambda C D M$ expectation. (Lower) Constraint on $r$ derived from the cleaned spectrum compared to the fiducial analysis shown in Fig. 6.

the difference spectra, and marginalize over $A_{d}$ and $\beta_{d}$, and hence $\alpha$, adopting the prior $\beta_{d}=1.59 \pm 0.11$. This alternative likelihood has the advantage of being less sensitive to non-Gaussianity of the dust, since only the 2-point function of the dust affects the covariance of the differenced spectra close to $\alpha_{\text {fid }}$, while the full analysis may, in principle, be affected by the non-Gaussianity of the dust through 4-point contributions to power spectra covariances. This cleaning approach does, however, ignore the (small amount of) additional information available at other frequencies. The lower panel of Fig. 12 compares the result to the fiducial analysis with the full multispectra likelihood. It is clear from the widths of the likelihood curves that compressing the spectra to form the cleaned difference results in very little loss of information on $r$. The difference in peak values arises from the different data 
treatments and is consistent with the scatter seen across simulations. Finally, we note that one could also form a combination $\left(\mathrm{BK} \times \mathrm{BK}-2 \alpha \mathrm{BK} \times \mathrm{P}+\alpha^{2} \mathrm{P} \times \mathrm{P}\right) /(1-\alpha)^{2}$ in which dust does not enter at all for $\alpha=\alpha_{\text {fid }}$. However, the variance of this combination of spectra is large due to the Planck noise levels, and likelihoods built from this combination are considerably less constraining.

\section{POSSIBLE CAUSES OF DECORRELATION}

Any systematic error that suppresses the BK150 $\times$ P353 cross-frequency spectrum with respect to the BK150 $\times$ BK150 and P353 $\times$ P353 single-frequency spectra would cause a systematic upward bias on the $r$ constraint. Here we investigate a couple of possibilities.

\section{A. Spatially varying dust frequency spectrum}

If the frequency dependence of polarized dust emission varied from place to place on the sky, it would cause the 150 and $353 \mathrm{GHz}$ dust sky patterns to decorrelate and suppress the BK150 $\times$ P353 cross-frequency spectrum relative to the single-frequency spectra. The assumption made so far in this Letter is that such decorrelation is negligible. In fact, PIP-XXX implicitly tests for such variation in their Fig. 6, where the Planck single- and cross-frequency spectra are compared to the modified blackbody model (with the cross-frequency spectra plotted at the geometric mean of their respective frequencies). This plot is for an average over a large region of low foreground sky (24\%); however, note that if there were spatial variation of the spectral behavior anywhere in this region it would cause suppression of the cross-frequency spectra with respect to the single-frequency spectra.

PIP-XXX also tests explicitly for evidence of decorrelation of the dust pattern across frequencies. Their Fig. E.1 shows the results for large and small sky patches. The signal-to-noise ratio is low in clean regions, but no evidence of decorrelation is found.

As a further check, we artificially suppress the amplitude of the BK150 $\times$ P353 spectra in the Gaussian dust-only simulations (see Sec. IVA) by a conservative 10\% (PIPXXX sets a $7 \%$ upper limit). We find that the maximum likelihood value for $r$ shifts up by an average of 0.018 , while $A_{d}$ shifts down by an average of $0.43 \mu \mathrm{K}^{2}$, with the size of the shift proportional to the magnitude of the dust power in each given realization. This behavior is readily understandable-since the BK150 $\times$ BK150 and BK150 $\times$ P353 spectra dominate the statistical weight, a decrease of the latter is interpreted as a reduction in dust power, which is compensated by an increase in $r$. The bias on $r$ will be linearly related to the assumed decorrelation factor.

\section{B. Calibration, analysis, etc.}

Figure 3 shows that the $E E$ spectrum $\mathrm{BK} 150 \times \mathrm{BK} 150$ is extremely similar to that for $\mathrm{BK} 150 \times \mathrm{P} 143$. We can compare such spectra to set limits on possible decorrelation between the BICEP2/Keck and Planck maps arising from any instrumental or analysis related effect, including differential pointing, polarization angle mischaracterization, etc. Taking the ratio of BK150 $\times$ P143 to the geometric mean of $\mathrm{BK} 150 \times \mathrm{BK} 150$ and $\mathrm{P} 143 \mathrm{H} 1 \times \mathrm{P} 143 \mathrm{H} 2$, we find that for $T T$ the decorrelation is approximately $0.1 \%$. For $E E$ the signal-to-noise ratio is lower, but decorrelation is limited to below $2 \%$, and consistent with zero when compared to the fluctuation of signal + noise simulations.

\section{CONCLUSIONS}

BK-I reported a highly significant detection of $B$-mode polarization, at $150 \mathrm{GHz}$, in excess of the lensed- $\Lambda \mathrm{CDM}$ expectation over the range $30<\ell<150$. This excess has been confirmed by additional data on the same field from the successor experiment Keck Array. PIP-XXX found that the level of dust power in a field centered on the BICEP2/Keck region (but somewhat larger than it) is of the same magnitude as the reported excess, but noted that, "the present uncertainties are large," and that a joint analysis was required.

In this Letter we have performed this joint analysis, using the combined BICEP $2 /$ Keck maps. Cross-correlating these maps against all of the polarized frequency bands of Planck we find a highly significant $B$-mode detection only in the cross spectrum with $353 \mathrm{GHz}$. We emphasize that this $150 \times 353 \mathrm{GHz}$ cross spectrum has a much higher signal-to-noise ratio than the $353 \mathrm{GHz}$ single-frequency spectrum that PIP-XXX analyzed.

We have analyzed the data using a multifrequency, multicomponent fit. In this fit it is necessary to impose a prior on the variation of the brightness of the polarized dust emission with observing frequency, since the available data are unable to constrain this alone, due to the relatively low signal-to-noise ratio in $B$-mode polarization at $353 \mathrm{GHz}$. However, based on the available information from Planck on the frequency dependence of polarized dust emission across the mid- and high-Galactic latitude sky, and the patch-to-patch stability thereof, this prior appears to be justified and conservative.

We have shown that the final constraint on the tensor-toscalar ratio $r$ is very stable when varying the frequency bands used, as well as the model priors. The result does differ when using the BICEP2 and Keck Array data alone rather than in combination, but the difference is compatible with noise fluctuation. Expanding the model to include synchrotron emission, while also including lower Planck frequencies, does not change the result.

Allowing the amplitude of lensing to be free, we obtain $A_{L}=1.13 \pm 0.18$, with a significance of detection of $7.0 \sigma$. This is the most significant direct detection to date of lensing in $B$-mode polarization, even compared to experiments with higher angular resolution. The POLARBEAR experiment has reported a detection of $B$-mode lensing 
on smaller angular scales $(500<\ell<2100)$, rejecting the $A_{L}=0$ hypothesis at $97.2 \%$ confidence [46]. Additionally, ACT [47] and SPT [48] have reported lensing detections in polarization in cross-correlation with some other tracer of the dark matter distribution on the sky.

We have validated the main likelihood analysis on simulations of a dust-only model and performed a simple subtraction of scaled spectra, which approximates a mapbased dust cleaning (obtaining an $r$ constraint curve that peaks somewhat lower). Finally, we investigated the possibility of astrophysical or instrumental decorrelation of the sky patterns between experiments or frequencies and find no evidence for relevant bias.

The final result is expressed as a likelihood curve for $r$, and yields an upper limit $r<0.12$ at $95 \%$ confidence. The median limit in the lensed- $\Lambda \mathrm{CDM}+$ noise + dust simulations is $r<0.075$. It is interesting to compare this latter to dust-free simulations using only BICEP2/Keck where the median limit is $r<0.03$ - the difference represents the limitation due to noise in the Planck maps, when marginalizing over dust. The $r$ constraint curve peaks at $r=0.05$ but disfavors zero only by a factor of 2.5 . This is expected by chance $8 \%$ of the time, as confirmed in simulations of a dust-only model. We emphasize that this significance is too low to be interpreted as a detection of primordial $B$ modes. Transforming the Planck temperature-only $95 \%$ confidence limit of $r_{0.002}<0.11$ [3] to the pivot scale used in this Letter yields $r_{0.05}<0.12$, compatible with the present result.

A COSMOMC module containing the band powers for all cross spectra between the combined BICEP2/Keck maps and all of the frequencies of Planck is available for download in Ref. [49].

In order to further constrain or detect IGW, additional data are required. The Planck Collaboration may be able to make progress alone using the large angular scale "reionization bump," if systematics can be appropriately controlled [50]. To take small patch "recombination bump" studies of type pursued here to the next level, data with signal-to-noise comparable to that achieved by BICEP2/Keck at $150 \mathrm{GHz}$ are required at more than one frequency. Figure 13 summarizes the situation. The BICEP2/Keck noise is much lower in the BICEP2/Keck field than the Planck noise. However, since dust emission is dramatically brighter at $353 \mathrm{GHz}$, it is detected in the cross spectrum between BICEP2/Keck and Planck $353 \mathrm{GHz}$. Synchrotron is not detected and the crossover frequency with dust is $\lesssim 100 \mathrm{GHz}$. Planck's PR2 data release [51] shows that for the cleanest $73 \%$ of the sky, at 40 arc min scales, the polarized foreground minimum is at 80-90 GHz. During the 2014 season, two of the Keck Array receivers observed in the $95 \mathrm{GHz}$ band and these data are under active analysis. BICEP3 will add substantial additional sensitivity at $95 \mathrm{GHz}$ in the 2015 , and especially 2016, seasons. Meanwhile, many other ground-based and

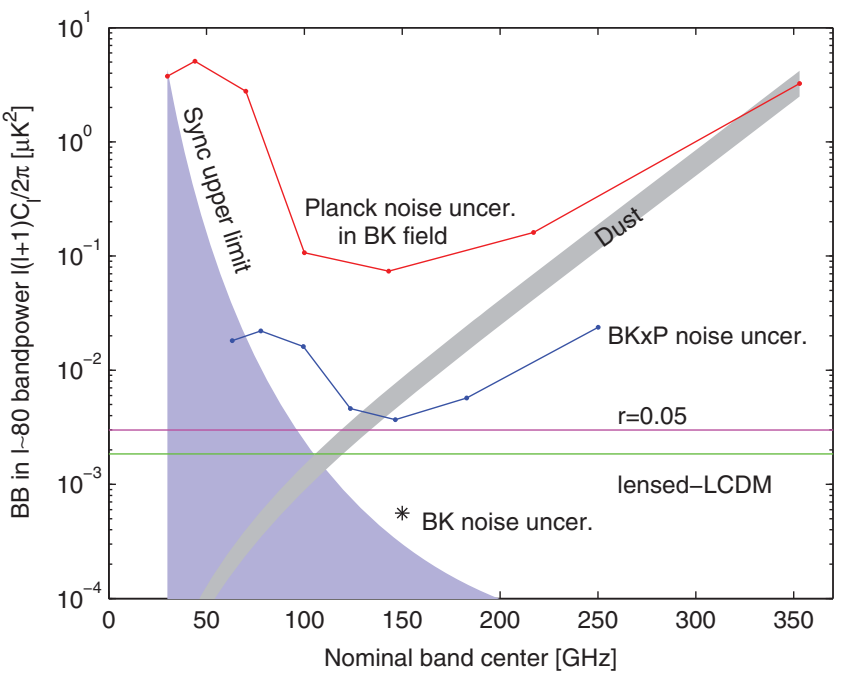

FIG. 13 (color). Expectation values, and uncertainties thereon, for the $\ell \sim 80 \mathrm{BB}$ band power in the BICEP2/Keck field. The green and magenta lines correspond to the expected signal power of lensed $\Lambda \mathrm{CDM}$ and $r=0.05$. Since CMB units are used, the levels corresponding to these are flat with frequency. The gray band shows the best fit dust model (see Sec. III B) and the blue shaded region shows the allowed region for synchrotron (see Sec. III C). The BICEP2/Keck noise uncertainty is shown as a single starred point, and the noise uncertainties of the Planck single-frequency spectra evaluated in the BICEP2/Keck field are shown in red. The blue points show the noise uncertainty of the cross spectra taken between BICEP2/Keck and, from left to right, Planck 30, 44, 70, 100, 143, 217, and $353 \mathrm{GHz}$, and plotted at horizontal positions such that they can be compared vertically with the dust and sync curves.

suborbital experiments are making measurements at a variety of frequencies and sky coverage fractions.

\section{ACKNOWLEDGMENTS}

BICEP2 was supported by the U.S. National Science Foundation under Grants No. ANT-0742818 and No. ANT1044978 (Caltech and Harvard) and No. ANT-0742592 and No. ANT-1110087 (Chicago and Minnesota). The development of antenna-coupled detector technology was supported by the JPL Research and Technology Development Fund and Grants No. 06-ARPA206-0040 and No. 10SAT10-0017 from the NASA APRA and SAT programs. The Keck Array project was supported by the National Science Foundation under Grants No. ANT-1145172 (Harvard), No. ANT-1145143 (Minnesota) and No. ANT-1145248 (Stanford), and from the Keck Foundation (Caltech). We thank the staff of the U.S. Antarctic Program and in particular the South Pole Station without whose help this research would not have been possible. The Planck Collaboration acknowledges the support of the following: ESA; CNES, and CNRS/INSUIN2P3-INP (France); ASI, CNR, and INAF (Italy); NASA and DoE (USA); STFC and UKSA (UK); CSIC, MINECO, 
JA and RES (Spain); Tekes, AoF, and CSC (Finland); DLR and MPG (Germany); CSA (Canada); DTU Space (Denmark); SER/SSO (Switzerland); RCN (Norway); SFI (Ireland); FCT/MCTES (Portugal); ERC and PRACE (EU). A description of the Planck Collaboration and a list of its members, indicating which technical or scientific activities they have been involved in, can be found in Ref. [52].

[1] A. A. Penzias and R. W. Wilson, A measurement of excess antenna temperature at $4080 \mathrm{Mc} / \mathrm{s}$, Astrophys. J. 142, 419 (1965).

[2] C. L. Bennett, D. Larson, J. L. Weiland, N. Jarosik, G. Hinshaw, N. Odegard, K. M. Smith, R. S. Hill, B. Gold, M. Halpern, E. Komatsu, M. R. Nolta, L. Page, D. N. Spergel, E. Wollack, J. Dunkley, A. Kogut, M. Limon, S. S. Meyer, G. S. Tucker, and E. L. Wright, Nine-year Wilkinson Microwave Anisotropy Probe (WMAP) observations: Final maps and results, Astrophys. J. Suppl. Ser. 208, 20 (2013).

[3] Planck Collaboration XVI, Cosmological parameters, Astron. Astrophys. 571, A16 (2014).

[4] Planck Collaboration XXII, Constraints on inflation, Astron. Astrophys. 571, A22 (2014).

[5] A. A. Starobinsky, Zh. Eksp. Teor. Fiz. Pisma Red. 30, 719 (1979).

[6] V. A. Rubakov, M. V. Sazhin, and A. V. Veryaskin, Graviton creation in the inflationary universe and the grand unification scale, Phys. Lett. B 115, 189 (1982).

[7] R. Fabbri and M. D. Pollock, The effect of primordially produced gravitons upon the anisotropy of the cosmological microwave background radiation, Phys. Lett. B 125, 445 (1983).

[8] L. F. Abbott and M. B. Wise, Constraints on generalized inflationary cosmologies, Nucl. Phys. B244, 541 (1984).

[9] A. G. Polnarev, Sov. Astron. 29, 607 (1985).

[10] U. Seljak and M. Zaldarriaga, Signature of Gravity Waves in Polarization of the Microwave Background, Phys. Rev. Lett. 78, 2054 (1997).

[11] U. Seljak, Measuring polarization in cosmic microwave background, Astrophys. J. 482, 6 (1997).

[12] M. Kamionkowski, A. Kosowsky, and A. Stebbins, A Probe of Primordial Gravity Waves and Vorticity, Phys. Rev. Lett. 78, 2058 (1997).

[13] Planck (http://www.esa.int/planck) is a project of the European Space Agency (ESA) with instruments provided by two scientific consortia funded by ESA member states (in particular the lead countries, France and Italy) with contributions from NASA (USA), and telescope reflectors provided in a collaboration between ESA and a scientific consortium led and funded by Denmark.

[14] Planck Collaboration 2011A, The Planck mission, Astron. Astrophys. 536, A1 (2011).

[15] Planck Collaboration I, Overview of products and scientific results, Astron. Astrophys. 571, A1 (2014).

[16] R. H. Hildebrand, J. L. Dotson, C. D. Dowell, D. A. Schleuning, and J. E. Vaillancourt, The far-infrared polarization spectrum: First results and analysis, Astrophys. J. 516, 834 (1999).
[17] B. T. Draine, Astrophysics of dust in cold clouds, in The Cold Universe, Saas-Fee Advanced Course 32, Lecture Notes 2002 of the Swiss Society for Astronomy and Astrophysics (SSAA), edited by A. W. Blain, F. Combes, B. T. Draine, D. Pfenniger, and Y. Revaz (Springer-Verlag, Berlin, 2004), p. 213, astro-ph/0304488.

[18] P. G. Martin, On predicting the polarization of low frequency emission by diffuse interstellar dust, in EAS Publications Series, edited by M.-A. Miville-Deschênes and F. Boulanger (EAS Publications, Paris, 2007), Vol. 23, pp. $165-188$.

[19] A. Benoît, P. Ade, A. Amblard, R. Ansari, É. Aubourg, S. Bargot, J. G. Bartlett, J.-P. Bernard, R. S. Bhatia, A. Blanchard, J. J. Bock, A. Boscaleri, F. R. Bouchet, A. Bourrachot, P. Camus, F. Couchot, P. de Bernardis, J. Delabrouille, F.-X. Désert, O. Doré et al., First detection of polarization of the submillimetre diffuse galactic dust emission by Archeops, Astron. Astrophys. 424, 571 (2004).

[20] L. Page, G. Hinshaw, E. Komatsu, M. R. Nolta, D. N. Spergel, C. L. Bennett, C. Barnes, R. Bean, O. Doré, J. Dunkley, M. Halpern, R. S. Hill, N. Jarosik, A. Kogut, M. Limon, S. S. Meyer, N. Odegard, H. V. Peiris, G. S. Tucker, L. Verde et al., Three year Wilkinson Microwave Anisotropy Probe (WMAP) observations: Polarization analysis, Astrophys. J. Suppl. Ser. 170, 335 (2007).

[21] BICEP2 Collaboration II, Experiment and three-year data set, Astrophys. J. 792, 62 (2014).

[22] BICEP2 Collaboration I, Detection of B-Mode Polarization at Degree Angular Scales, Phys. Rev. Lett. 112, 241101 (2014).

[23] J. Dunkley, A. Amblard, C. Baccigalupi, M. Betoule, D. Chuss, A. Cooray, J. Delabrouille, C. Dickinson, G. Dobler, J. Dotson, H. K. Eriksen, D. Finkbeiner, D. Fixsen, P. Fosalba, A. Fraisse, C. Hirata, A. Kogut, J. Kristiansen, C. Lawrence, A. M. Magalhães et al., Prospects for polarized foreground removal, AIP Conf. Proc. 1141, 222 (2009).

[24] J. Delabrouille, M. Betoule, J.-B. Melin, M.-A. MivilleDeschênes, J. Gonzalez-Nuevo, M. Le Jeune, G. Castex, G. de Zotti, S. Basak, M. Ashdown, J. Aumont, C. Baccigalupi, A. J. Banday, J.-P. Bernard, F. R. Bouchet, D. L. Clements, A. da Silva, C. Dickinson, F. Dodu, K. Dolag et al., The prelaunch Planck Sky Model: A model of sky emission at submillimetre to centimetre wavelengths, Astron. Astrophys. 553, A96 (2013).

[25] Planck Collaboration Int. XIX, An overview of the polarized thermal emission from Galactic dust, arXiv:1405.0871v1.

[26] Planck Collaboration Int. XXII, Frequency dependence of thermal emission from Galactic dust in intensity and polarization, arXiv:1405.0874.

[27] R. Flauger, J. C. Hill, and D. N. Spergel, Toward an understanding of foreground emission in the BICEP2 region, J. Cosmol. Astropart. Phys. 8 (2014) 039.

[28] M. J. Mortonson and U. Seljak, A joint analysis of Planck and BICEP2 B modes including dust polarization uncertainty, J. Cosmol. Astropart. Phys. 10 (2014) 035.

[29] Planck Collaboration Int. XXX, The angular power spectrum of polarized dust emission at intermediate and high Galactic latitudes, arXiv:1409.5738. 
[30] Keck Array and BICEP2 Collaborations V, Measurements of B-mode polarization at degree angular scales and $150 \mathrm{GHz}$ by the Keck array, arXiv:1502.00643.

[31] http://archives.esac.esa.int/pla2.

[32] Planck Collaboration 2015-I, Overview of products and scientific results, arXiv:1502.01582.

[33] Planck Collaboration 2015-XII, Simulations (to be published).

[34] Planck Collaboration IV, Low Frequency Instrument beams and window functions, Astron. Astrophys. 571, A4 (2014).

[35] Planck Collaboration VII, HFI time response and beams, Astron. Astrophys. 571, A7 (2014).

[36] http://healpix.sourceforge.net/.

[37] K. M. Górski, E. Hivon, A. J. Banday, B. D. Wandelt, F. K. Hansen, M. Reinecke, and M. Bartelmann, HEALPix: A framework for high-resolution discretization and fast analysis of data distributed on the sphere, Astrophys. J. 622, 759 (2005).

[38] With parameters taken from Planck [3].

[39] M. Tristram, J. F. Macías-Pérez, C. Renault, and D. Santos, Xspect, estimation of the angular power spectrum by computing cross-power spectra with analytical error bars, Mon. Not. R. Astron. Soc. 358, 833 (2005).

[40] M. Preece, Analysis of Cosmic Microwave Background Polarisation, Ph.D. thesis, The University of Manchester, U.K., 2011.

[41] S. Hamimeche and A. Lewis, Likelihood analysis of CMB temperature and polarization power spectra, Phys. Rev. D 77, 103013 (2008).

[42] D. Barkats, R. Aikin, C. Bischoff, I. Buder, J. P. Kaufman, B. G. Keating, J. M. Kovac, M. Su, P. A. R. Ade, J. O. Battle, E. M. Bierman, J. J. Bock, H. C. Chiang, C. D. Dowell, L. Duband, J. Filippini, E. F. Hivon, W. L. Holzapfel, V. V. Hristov, W. C. Jones et al., Degree-scale cosmic microwave background polarization measurements from three years of BICEP1 data, Astrophys. J. 783, 67 (2014).
[43] A. Lewis and S. Bridle, Cosmological parameters from CMB and other data: A Monte Carlo approach, Phys. Rev. D 66, 103511 (2002).

[44] Planck Collaboration Int. XVII, Emission of dust in the diffuse interstellar medium from the far-infrared to microwave frequencies, Astron. Astrophys. 566, A55 (2014).

[45] Note that this is the number evaluated at $353 \mathrm{GHz}$ exactlythe equivalent number as integrated over the Planck $353 \mathrm{GHz}$ passband is $4.5 \mu \mathrm{K}^{2}$ and the mask used in PIP-XXX is somewhat different (larger) than the BICEP2/Keck mask used here.

[46] POLARBEAR Collaboration, A measurement of the cosmic microwave background B-mode polarization power spectrum at sub-degree scales with POLARBEAR, Astrophys. J. 794, 171 (2014).

[47] A. van Engelen, B. D. Sherwin, N. Sehgal, G. E. Addison, R. Allison, N. Battaglia, F. de Bernardis, E. Calabrese, K. Coughlin, D. Crichton, J. R. Bond, R. Datta, R. Dunner, J. Dunkley, E. Grace, M. Gralla, A. Hajian, M. Hasselfield, S. Henderson, J. C. Hill et al., The Atacama Cosmology Telescope: Lensing of CMB temperature and polarization derived from cosmic infrared background cross-correlation, arXiv:1412.0626.

[48] D. Hanson, S. Hoover, A. Crites, P. A. R. Ade, K. A. Aird, J. E. Austermann, J. A. Beall, A. N. Bender, B. A. Benson, L. E. Bleem, J. J. Bock, J. E. Carlstrom, C. L. Chang, H. C. Chiang, H.-M. Cho, A. Conley, T. M. Crawford, T. de Haan, M. A. Dobbs, W. Everett et al., Detection of B-Mode Polarization in the Cosmic Microwave Background with Data from the South Pole Telescope, Phys. Rev. Lett. 111, 141301 (2013).

[49] http://bicepkeck.org.

[50] G. Efstathiou and S. Gratton, B-mode detection with an extended Planck mission, J. Cosmol. Astropart. Phys. 6 (2009) 011.

[51] Planck Collaboration 2015-X, Diffuse component separation: Foreground maps, arxiv:1502.01588.

[52] http://www.cosmos.esa.int/web/planck/planck-collaboration.

P. A. R. Ade, ${ }^{1}$ N. Aghanim, ${ }^{2}$ Z. Ahmed, ${ }^{3}$ R. W. Aikin, ${ }^{4}$ K. D. Alexander, ${ }^{5}$ M. Arnaud, ${ }^{6}$ J. Aumont, ${ }^{2}$ C. Baccigalupi ${ }^{7}$ A. J. Banday,${ }^{8,9}$ D. Barkats, ${ }^{10}$ R. B. Barreiro, ${ }^{11}$ J. G. Bartlett, ${ }^{12,13}$ N. Bartolo, ${ }^{14,15}$ E. Battaner,${ }^{16,17}$ K. Benabed, ${ }^{18,19}$ A. Benoît, ${ }^{20}$ A. Benoit-Lévy, ${ }^{21,18,19}$ S. J. Benton, ${ }^{22}$ J.-P. Bernard, ${ }^{8,9}$ M. Bersanelli, ${ }^{23,24}$ P. Bielewicz, ${ }^{8,9,7}$ C. A. Bischoff, ${ }^{5}$ J. J. Bock, ${ }^{13,4}$ A. Bonaldi, ${ }^{25}$ L. Bonavera, ${ }^{11}$ J. R. Bond, ${ }^{26}$ J. Borrill, ${ }^{27,28}$ F. R. Bouchet,${ }^{18,29}$ F. Boulanger, ${ }^{2}$ J. A. Brevik, ${ }^{4}$ M. Bucher, ${ }^{12}$ I. Buder, ${ }^{5}$ E. Bullock, ${ }^{30}$ C. Burigana, ${ }^{31,32,33}$ R. C. Butler, ${ }^{31}$ V. Buza, ${ }^{5}$ E. Calabrese, ${ }^{34}$ J.-F. Cardoso, ${ }^{35,12,18}$ A. Catalano, ${ }^{36,37}$ A. Challinor, ${ }^{38,39,40}$ R.-R. Chary, ${ }^{41}$ H. C. Chiang, ${ }^{42,43}$ P. R. Christensen, ${ }^{44,45}$ L. P. L. Colombo, ${ }^{46,13}$ C. Combet, ${ }^{36}$ J. Connors, ${ }^{5}$ F. Couchot, ${ }^{47}$ A. Coulais,,${ }^{37}$ B. P. Crill,${ }^{13,4}$ A. Curto, ${ }^{48,11}$ F. Cuttaia, ${ }^{31}$ L. Danese, ${ }^{7}$ R. D. Davies, ${ }^{25}$ R. J. Davis, ${ }^{25}$ P. de Bernardis, ${ }^{49}$ A. de Rosa, ${ }^{31}$ G. de Zotti, ${ }^{50,7}$ J. Delabrouille, ${ }^{12}$ J.-M. Delouis, ${ }^{18,19}$ F.-X. Désert, ${ }^{51}$ C. Dickinson, ${ }^{25}$ J. M. Diego,${ }^{11}$ H. Dole, ${ }^{2,52}$ S. Donzelli, ${ }^{24}$ O. Doré,${ }^{13,4}$ M. Douspis, ${ }^{2}$ C. D. Dowell, ${ }^{13}$ L. Duband,${ }^{53}$ A. Ducout ${ }^{18,54}$ J. Dunkley, ${ }^{34}$ X. Dupac,${ }^{55}$ C. Dvorkin, ${ }^{5}$ G. Efstathiou, ${ }^{38}$ F. Elsner, ${ }^{21,18,19}$ T. A. Enßlin, ${ }^{56}$ H. K. Eriksen, ${ }^{57}$ E. Falgarone, ${ }^{37}$ J. P. Filippini, ${ }^{4,58}$ F. Finelli, ${ }^{31,33}$ S. Fliescher, ${ }^{59}$ O. Forni,${ }^{8,9}$ M. Frailis, ${ }^{60}$ A. A. Fraisse, ${ }^{42}$ E. Franceschi, ${ }^{31}$ A. Frejsel, ${ }^{44}$ S. Galeotta, ${ }^{60}$ S. Galli, ${ }^{18}$ K. Ganga, ${ }^{12}$ T. Ghosh, ${ }^{2}$ M. Giard, ${ }^{8,9}$ E. Gjerløw, ${ }^{57}$ S. R. Golwala, ${ }^{4}$ J. González-Nuevo, ${ }^{11,7}$ K. M. Górski, ${ }^{13,61}$ S. Gratton, ${ }^{39,38}$ A. Gregorio, ${ }^{62,60,63}$ A. Gruppuso, ${ }^{31}$ J. E. Gudmundsson, ${ }^{42}$ M. Halpern, ${ }^{64}$ F. K. Hansen, ${ }^{57}$ D. Hanson, ${ }^{65,13,26}$ D. L. Harrison, ${ }^{38,39}$ M. Hasselfield, ${ }^{64}$ G. Helou, ${ }^{4}$ S. Henrot-Versillé, ${ }^{47}$ D. Herranz, ${ }^{11}$ S. R. Hildebrandt, ${ }^{13,4}$ G. C. Hilton, ${ }^{66}$ E. Hivon, ${ }^{18,19}$ M. Hobson, ${ }^{48}$ W. A. Holmes, ${ }^{13}$ W. Hovest,${ }^{56}$ V. V. Hristov, ${ }^{4}$ 
K. M. Huffenberger, ${ }^{67}$ H. Hui, ${ }^{4}$ G. Hurier, ${ }^{2}$ K. D. Irwin, ${ }^{3,68,66}$ A. H. Jaffe, ${ }^{54}$ T. R. Jaffe, ${ }^{8,9}$ J. Jewell, ${ }^{13}$ W. C. Jones, ${ }^{42}$ M. Juvela, ${ }^{69}$ A. Karakci, ${ }^{12}$ K. S. Karkare, ${ }^{5}$ J. P. Kaufman, ${ }^{70}$ B. G. Keating, ${ }^{70}$ S. Kefeli, ${ }^{4}$ E. Keihänen, ${ }^{69}$ S. A. Kernasovskiy, ${ }^{3}$ R. Keskitalo, ${ }^{27}$ T. S. Kisner, ${ }^{71}$ R. Kneissl, ${ }^{72,73}$ J. Knoche, ${ }^{56}$ L. Knox, ${ }^{74}$ J. M. Kovac, ${ }^{5}$ N. Krachmalnicoff, ${ }^{23}$ M. Kunz, ${ }^{75,2,76}$ C. L. Kuo, ${ }^{3,68}$ H. Kurki-Suonio, ${ }^{69,77}$ G. Lagache, ${ }^{78,2}$ A. Lähteenmäki, ${ }^{79,77}$ J.-M. Lamarre, ${ }^{37}$ A. Lasenby, ${ }^{48,39}$ M. Lattanzi, ${ }^{32}$ C. R. Lawrence, ${ }^{13}$ E. M. Leitch, ${ }^{80}$ R. Leonardi, ${ }^{55}$ F. Levrier, ${ }^{37}$ A. Lewis, ${ }^{81}$ M. Liguori, ${ }^{14,15}$ P. B. Lilje,${ }^{57}$ M. Linden-Vørnle, ${ }^{82}$ M. López-Caniego, ${ }^{55,11}$ P. M. Lubin,${ }^{83}$ M. Lueker, ${ }^{4}$ J. F. Macías-Pérez, ${ }^{36}$ B. Maffei, ${ }^{25}$ D. Maino, ${ }^{23,24}$ N. Mandolesi, ${ }^{31,32}$ A. Mangilli, ${ }^{2,47}$ M. Maris, ${ }^{60}$ P. G. Martin, ${ }^{26}$ E. Martínez-González, ${ }^{11}$ S. Masi ${ }^{49}$ P. Mason, ${ }^{4}$ S. Matarrese,${ }^{14,15,84}$ K. G. Megerian, ${ }^{13}$ P. R. Meinhold, ${ }^{83}$ A. Melchiorri, ${ }^{49,85}$ L. Mendes, ${ }^{55}$ A. Mennella, ${ }^{23,24}$ M. Migliaccio, ${ }^{38,39}$ S. Mitra, ${ }^{86,13}$ M.-A. Miville-Deschênes, ${ }^{2,26}$ A. Moneti, ${ }^{18}$ L. Montier, ${ }^{8,9}$ G. Morgante,${ }^{31}$ D. Mortlock, ${ }^{54}$ A. Moss, ${ }^{87}$ D. Munshi, ${ }^{1}$ J. A. Murphy, ${ }^{88}$ P. Naselsky, ${ }^{44,45}$ F. Nati, ${ }^{42}$ P. Natoli, ${ }^{32,89,31}$ C. B. Netterfield, ${ }^{90}$ H. T. Nguyen, ${ }^{13}$ H. U. Nørgaard-Nielsen, ${ }^{82}$ F. Noviello, ${ }^{25}$ D. Novikov, ${ }^{91}$ I. Novikov, ${ }^{44,91}$ R. O'Brient, ${ }^{13}$ R. W. Ogburn IV,${ }^{3,68}$ A. Orlando, ${ }^{70}$ L. Pagano, ${ }^{49,85}$ F. Pajot, ${ }^{2}$ R. Paladini, ${ }^{41}$ D. Paoletti, ${ }^{31,33}$ B. Partridge, ${ }^{92}$ F. Pasian, ${ }^{60}$ G. Patanchon, ${ }^{12}$ T. J. Pearson, ${ }^{4,41}$ O. Perdereau, ${ }^{47}$ L. Perotto, ${ }^{36}$ V. Pettorino, ${ }^{93}$ F. Piacentini, ${ }^{49}$ M. Piat, ${ }^{12}$ D. Pietrobon, ${ }^{13}$ S. Plaszczynski, ${ }^{47}$ E. Pointecouteau, ${ }^{8,9}$ G. Polenta,${ }^{89,94}$ N. Ponthieu, ${ }^{2,51}$ G. W. Pratt, ${ }^{6}$ S. Prunet, ${ }^{18,19, *}$ C. Pryke, ${ }^{59,30}$ J.-L. Puget, ${ }^{2}$ J. P. Rachen, ${ }^{95,56}$ W. T. Reach, ${ }^{96}$ R. Rebolo,,${ }^{97,98,99}$ M. Reinecke, ${ }^{56}$ M. Remazeilles, ${ }^{25,2,12}$ C. Renault, ${ }^{36}$ A. Renzi, ${ }^{100,101}$ S. Richter, ${ }^{5}$ I. Ristorcelli, ${ }^{8,9}$ G. Rocha, ${ }^{13,4}$ M. Rossetti, ${ }^{23,24}$ G. Roudier, ${ }^{12,37,13}$ M. Rowan-Robinson, ${ }^{54}$ J. A. Rubiño-Martín, ${ }^{97,99}$ B. Rusholme, ${ }^{41}$ M. Sandri, ${ }^{31}$ D. Santos, ${ }^{36}$ M. Savelainen,${ }^{69,77}$ G. Savini, ${ }^{102}$ R. Schwarz, ${ }^{59}$ D. Scott,${ }^{64}$ M. D. Seiffert, ${ }^{13,4}$ C. D. Sheehy, ${ }^{59,103}$ L. D. Spencer, ${ }^{1}$ Z. K. Staniszewski, ${ }^{4,13}$ V. Stolyarov, ${ }^{48,39,104}$ R. Sudiwala, ${ }^{1}$ R. Sunyaev, ${ }^{56,105}$ D. Sutton, ${ }^{38,39}$ A.-S. Suur-Uski, ${ }^{69,77}$ J.-F. Sygnet, ${ }^{18}$ J. A. Tauber, ${ }^{106}$ G. P. Teply, ${ }^{4}$ L. Terenzi,${ }^{107,31}$ K. L. Thompson, ${ }^{3}$ L. Toffolatti, ${ }^{108,11,31}$ J. E. Tolan, ${ }^{3}$ M. Tomasi ${ }^{23,24}$ M. Tristram, ${ }^{47}$ M. Tucci, ${ }^{75}$ A. D. Turner, ${ }^{13,80}$ L. Valenziano, ${ }^{31}$ J. Valiviita, ${ }^{69,77}$ B. Van Tent, ${ }^{109}$ L. Vibert, ${ }^{2}$ P. Vielva, ${ }^{11}$ A. G. Vieregg, ${ }^{103,110}$ F. Villa, ${ }^{31}$ L. A. Wade, ${ }^{13}$ B. D. Wandelt,,${ }^{18,19,58}$ R. Watson, ${ }^{25}$ A. C. Weber, ${ }^{13}$ I. K. Wehus,${ }^{13}$ M. White, ${ }^{111}$ S. D. M. White, ${ }^{56}$ J. Willmert, ${ }^{59}$ C. L. Wong, ${ }^{5}$ K. W. Yoon, ${ }^{3,68}$ D. Yvon, ${ }^{112}$ A. Zacchei, ${ }^{60}$ and A. Zonca ${ }^{83}$

\section{(BICEP2/Keck and Planck Collaborations)}

${ }^{1}$ School of Physics and Astronomy, Cardiff University, Queens Buildings, The Parade, Cardiff, CF24 3AA, United Kingdom ${ }^{2}$ Institut d'Astrophysique Spatiale, CNRS (UMR8617) Université Paris-Sud 11, Bâtiment 121, Orsay, France ${ }^{3}$ Department of Physics, Stanford University, Stanford, California 94305, USA

${ }^{4}$ California Institute of Technology, Pasadena, California, USA

${ }^{5}$ Harvard-Smithsonian Center for Astrophysics, 60 Garden Street MS 42, Cambridge, Massachusetts 02138, USA

${ }^{6}$ Laboratoire AIM, IRFU/Service d'Astrophysique-CEA/DSM-CNRS-Université Paris Diderot, Bâtiment 709, CEA-Saclay, F-91191 Gif-sur-Yvette Cedex, France

${ }^{7}$ SISSA, Astrophysics Sector, via Bonomea 265, 34136, Trieste, Italy

${ }^{8}$ Université de Toulouse, UPS-OMP, IRAP, F-31028 Toulouse cedex 4, France ${ }^{9}$ CNRS, IRAP, 9 Avenue colonel Roche, BP 44346, F-31028 Toulouse cedex 4, France

${ }^{10}$ Joint ALMA Observatory, Vitacura, Santiago, Chile

${ }^{11}$ Instituto de Física de Cantabria (CSIC-Universidad de Cantabria), Avenida de los Castros s/n, Santander, Spain

${ }^{12}$ APC, AstroParticule et Cosmologie, Université Paris Diderot, CNRS/IN2P3, CEA/lrfu, Observatoire de Paris, Sorbonne Paris Cité, 10, rue Alice Domon et Léonie Duquet, 75205 Paris Cedex 13, France

${ }^{13}$ Jet Propulsion Laboratory, California Institute of Technology, 4800 Oak Grove Drive, Pasadena, California, USA

${ }^{14}$ Dipartimento di Fisica e Astronomia G. Galilei, Università degli Studi di Padova, via Marzolo 8, 35131 Padova, Italy

${ }^{15}$ Istituto Nazionale di Fisica Nucleare, Sezione di Padova, via Marzolo 8, I-35131 Padova, Italy

${ }^{16}$ University of Granada, Departamento de Física Teórica y del Cosmos, Facultad de Ciencias, Granada, Spain

${ }^{17}$ University of Granada, Instituto Carlos I de Física Teórica y Computacional, Granada, Spain

${ }^{18}$ Institut d'Astrophysique de Paris, CNRS (UMR7095), 98 bis Boulevard Arago, F-75014, Paris, France

${ }^{19}$ UPMC Université de Paris 06, UMR7095, 98 bis Boulevard Arago, F-75014, Paris, France

${ }^{20}$ Institut Néel, CNRS, Université Joseph Fourier Grenoble I, 25 rue des Martyrs, Grenoble, France

${ }^{21}$ Department of Physics and Astronomy, University College London, London WC1E 6BT, United Kingdom

${ }^{22}$ Department of Physics, University of Toronto, Toronto, Ontario, M5S 1A7, Canada

${ }^{23}$ Dipartimento di Fisica, Università degli Studi di Milano, Via Celoria, 16, Milano, Italy

${ }^{24}$ INAF/IASF Milano, Via E. Bassini 15, Milano, Italy

${ }^{25}$ Jodrell Bank Centre for Astrophysics, Alan Turing Building, School of Physics and Astronomy, The University of Manchester, Oxford Road, Manchester, M13 9PL, United Kingdom

${ }^{26}$ CITA, University of Toronto, 60 St. George Street, Toronto, Ontario M5S 3H8, Canada

${ }^{27}$ Computational Cosmology Center, Lawrence Berkeley National Laboratory, Berkeley, California, USA 
${ }^{28}$ Space Sciences Laboratory, University of California, Berkeley, California, USA

${ }^{29}$ Sorbonne Université-UPMC, UMR7095, Institut d'Astrophysique de Paris, 98 bis Boulevard Arago, F-75014, Paris, France

${ }^{30}$ Minnesota Institute for Astrophysics, University of Minnesota, Minneapolis, Minnesota 55455, USA

${ }^{31}$ INAF/IASF Bologna, Via Gobetti 101, Bologna, Italy

${ }^{32}$ Dipartimento di Fisica e Scienze della Terra, Università di Ferrara, Via Saragat 1, 44122 Ferrara, Italy

${ }^{33}$ INFN, Sezione di Bologna, Via Irnerio 46, I-40126, Bologna, Italy

${ }^{34}$ Sub-Department of Astrophysics, University of Oxford, Keble Road, Oxford OX1 3RH, United Kingdom

${ }^{35}$ Laboratoire Traitement et Communication de l'Information, CNRS (UMR 5141) and Télécom ParisTech, 46 rue Barrault F-75634 Paris Cedex 13, France

${ }^{36}$ Laboratoire de Physique Subatomique et Cosmologie, Université Grenoble-Alpes, CNRS/IN2P3, 53, rue des Martyrs, 38026 Grenoble Cedex, France

${ }^{37}$ LERMA, CNRS, Observatoire de Paris, 61 Avenue de l'Observatoire, Paris, France

${ }^{38}$ Institute of Astronomy, University of Cambridge, Madingley Road, Cambridge CB3 OHA, United Kingdom

${ }^{39}$ Kavli Institute for Cosmology Cambridge, Madingley Road, Cambridge, CB3 OHA, United Kingdom

${ }^{40}$ Centre for Theoretical Cosmology, DAMTP, University of Cambridge, Wilberforce Road, Cambridge CB3 OWA, United Kingdom

${ }^{41}$ Infrared Processing and Analysis Center, California Institute of Technology, Pasadena, California 91125, USA

${ }^{42}$ Department of Physics, Princeton University, Princeton, New Jersey, USA

${ }^{43}$ Astrophysics \& Cosmology Research Unit, School of Mathematics, Statistics \& Computer Science, University of KwaZulu-Natal, Westville Campus, Private Bag X54001, Durban 4000, South Africa

${ }^{44}$ Niels Bohr Institute, Blegdamsvej 17, Copenhagen, Denmark

${ }^{45}$ Discovery Center, Niels Bohr Institute, Blegdamsvej 17, Copenhagen, Denmark

${ }^{46}$ Department of Physics and Astronomy, Dana and David Dornsife College of Letter, Arts and Sciences, University of Southern California, Los Angeles, California 90089, USA

${ }^{47}$ LAL, Université Paris-Sud, CNRS/IN2P3, Orsay, France

${ }^{48}$ Astrophysics Group, Cavendish Laboratory, University of Cambridge, J J Thomson Avenue, Cambridge CB3 OHE, United Kingdom

${ }^{49}$ Dipartimento di Fisica, Università La Sapienza, Piazzale Aldo Moro 2, Roma, Italy

${ }^{50}$ INAF-Osservatorio Astronomico di Padova, Vicolo dell'Osservatorio 5, Padova, Italy

${ }^{51}$ IPAG: Institut de Planétologie et d'Astrophysique de Grenoble, Université Grenoble Alpes, IPAG, F-38000 Grenoble, France, CNRS, IPAG, F-38000 Grenoble, France

${ }^{52}$ Institut Universitaire de France, 103, bd Saint-Michel, 75005, Paris, France

${ }^{53}$ Service des Basses Températures, Commissariat à l'Energie Atomique, 38054 Grenoble, France

${ }^{54}$ Imperial College London, Astrophysics group, Blackett Laboratory, Prince Consort Road, London, SW7 2AZ, United Kingdom

${ }^{55}$ European Space Agency, ESAC, Planck Science Office, Camino bajo del Castillo, s/n, Urbanización Villafranca del Castillo, Villanueva de la Cañada, Madrid, Spain

${ }^{56}$ Max-Planck-Institut für Astrophysik, Karl-Schwarzschild-Strasse 1, 85741 Garching, Germany

${ }^{57}$ Institute of Theoretical Astrophysics, University of Oslo, Blindern, Oslo, Norway

${ }^{58}$ Department of Physics, University of Illinois at Urbana-Champaign, 1110 West Green Street, Urbana, Illinois, USA

${ }^{59}$ School of Physics and Astronomy, University of Minnesota, Minneapolis, Minnesota 55455, USA

${ }^{60}$ INAF-Osservatorio Astronomico di Trieste, Via G.B. Tiepolo 11, Trieste, Italy

${ }^{61}$ Warsaw University Observatory, Aleje Ujazdowskie 4, 00-478 Warszawa, Poland

${ }^{62}$ Dipartimento di Fisica, Università degli Studi di Trieste, via Alfonso Valerio 2, Trieste, Italy

${ }^{63}$ INFN/National Institute for Nuclear Physics, Via Valerio 2, I-34127 Trieste, Italy

${ }^{64}$ Department of Physics \& Astronomy, University of British Columbia, 6224 Agricultural Road, Vancouver, British Columbia, Canada

${ }^{65}$ McGill Physics, Ernest Rutherford Physics Building, McGill University, 3600 rue University, Montréal, Quebec, H3A 2T8, Canada

${ }^{66}$ National Institute of Standards and Technology, Boulder, Colorado 80305, USA

${ }^{67}$ Department of Physics, Florida State University, Keen Physics Building, 77 Chieftan Way, Tallahassee, Florida, USA

${ }^{68}$ Kavli Institute for Particle Astrophysics and Cosmology, SLAC National Accelerator Laboratory, 2575 Sand Hill Road, Menlo Park, California 94025, USA

${ }^{69}$ Department of Physics, Gustaf Hällströmin katu 2a, University of Helsinki, Helsinki, Finland

${ }^{70}$ Department of Physics, University of California at San Diego, La Jolla, California 92093, USA

${ }^{71}$ Lawrence Berkeley National Laboratory, Berkeley, California, USA

${ }^{72}$ European Southern Observatory, ESO Vitacura, Alonso de Cordova 3107, Vitacura, Casilla 19001, Santiago, Chile

${ }^{73}$ Atacama Large Millimeter/submillimeter Array, ALMA Santiago Central Offices, Alonso de Cordova 3107, Vitacura, Casilla 763 0355, Santiago, Chile

${ }^{74}$ Department of Physics, University of California, One Shields Avenue, Davis, California, USA

${ }^{75}$ Département de Physique Théorique, Université de Genève, 24, Quai E. Ansermet, 1211 Genève 4, Switzerland

${ }^{76}$ African Institute for Mathematical Sciences, 6-8 Melrose Road, Muizenberg, Cape Town, South Africa

${ }^{77}$ Helsinki Institute of Physics, Gustaf Hällströmin katu 2, University of Helsinki, Helsinki, Finland

${ }^{78}$ Aix Marseille Université, CNRS, LAM (Laboratoire d'Astrophysique de Marseille) UMR 7326, 13388, Marseille, France 
${ }^{79}$ Aalto University Metsähovi Radio Observatory and Department of Radio Science and Engineering, P.O. Box 13000, FI-00076 AALTO, Finland

${ }^{80}$ University of Chicago, Chicago, Illinois 60637, USA

${ }^{81}$ Department of Physics and Astronomy, University of Sussex, Brighton BN1 9QH, United Kingdom

${ }^{82}$ DTU Space, National Space Institute, Technical University of Denmark, Elektrovej 327, DK-2800 Kongens Lyngby, Denmark

${ }^{83}$ Department of Physics, University of California, Santa Barbara, California, USA

${ }^{84}$ Gran Sasso Science Institute, INFN, viale F. Crispi 7, 67100L'Aquila, Italy

${ }^{85}$ INFN, Sezione di Roma 1, Università di Roma Sapienza, Piazzale Aldo Moro 2, 00185, Roma, Italy

${ }^{86}$ IUCAA, Post Bag 4, Ganeshkhind, Pune University Campus, Pune 411 007, India

${ }^{87}$ School of Physics and Astronomy, University of Nottingham, Nottingham NG7 2RD, United Kingdom

${ }^{88}$ National University of Ireland, Department of Experimental Physics, Maynooth, County Kildare, Ireland

${ }^{89}$ Agenzia Spaziale Italiana Science Data Center, Via del Politecnico snc, 00133, Roma, Italy

${ }^{90}$ Department of Astronomy and Astrophysics, University of Toronto, 50 Saint George Street, Toronto, Ontario, Canada

${ }^{91}$ Lebedev Physical Institute of the Russian Academy of Sciences,

Astro Space Centre, 84/32 Profsoyuznaya st., Moscow, GSP-7, 117997, Russia

${ }^{92}$ Haverford College Astronomy Department, 370 Lancaster Avenue, Haverford, Pennsylvania, USA

${ }^{93}$ HGSFP and University of Heidelberg, Theoretical Physics Department, Philosophenweg 16, 69120, Heidelberg, Germany

${ }^{94}$ INAF-Osservatorio Astronomico di Roma, via di Frascati 33, Monte Porzio Catone, Italy

${ }^{95}$ Department of Astrophysics/IMAPP, Radboud University Nijmegen, P.O. Box 9010, 6500 GL Nijmegen, The Netherlands

${ }^{96}$ Universities Space Research Association, Stratospheric Observatory for Infrared Astronomy, MS 232-11, Moffett Field, California 94035, USA

${ }^{97}$ Instituto de Astrofísica de Canarias, C/Vía Láctea s/n, La Laguna, Tenerife, Spain

${ }^{98}$ Consejo Superior de Investigaciones Científicas (CSIC), Madrid, Spain

${ }^{99}$ Departamento Astrofísica, Universidad de La Laguna (ULL), E-38206 La Laguna, Tenerife, Spain

${ }^{100}$ Dipartimento di Matematica, Università di Roma Tor Vergata, Via della Ricerca Scientifica, 1, Roma, Italy

${ }^{101}$ INFN, Sezione di Roma 2, Università di Roma Tor Vergata, Via della Ricerca Scientifica, 1, Roma, Italy

${ }^{102}$ Optical Science Laboratory, University College London, Gower Street, London, United Kingdom

${ }^{103}$ Kavli Institute for Cosmological Physics, University of Chicago, Chicago, Illinois 60637, USA

${ }^{104}$ Special Astrophysical Observatory, Russian Academy of Sciences, Nizhnij Arkhyz, Zelenchukskiy region, Karachai-Cherkessian Republic, 369167, Russia

${ }^{105}$ Space Research Institute (IKI), Russian Academy of Sciences, Profsoyuznaya Street, 84/32, Moscow, 117997, Russia

${ }^{106}$ European Space Agency, ESTEC, Keplerlaan 1, 2201 AZ Noordwijk, The Netherlands

${ }^{107}$ Facoltà di Ingegneria, Università degli Studi e-Campus, Via Isimbardi 10, Novedrate (CO), 22060, Italy

${ }^{108}$ Departamento de Física, Universidad de Oviedo, Avda. Calvo Sotelo s/n, Oviedo, Spain

${ }^{109}$ Laboratoire de Physique Théorique, Université Paris-Sud 11 \& CNRS, Bâtiment 210, 91405 Orsay, France

${ }^{110}$ Department of Physics, Enrico Fermi Institute, University of Chicago, Chicago, Illinois 60637, USA

${ }^{111}$ Department of Physics, University of California, Berkeley, California, USA

${ }^{112}$ DSM/Irfu/SPP, CEA-Saclay, F-91191 Gif-sur-Yvette Cedex, France

${ }^{\dagger}$ Brendan.P.Crill@jpl.nasa.gov

pryke@physics.umn.edu 